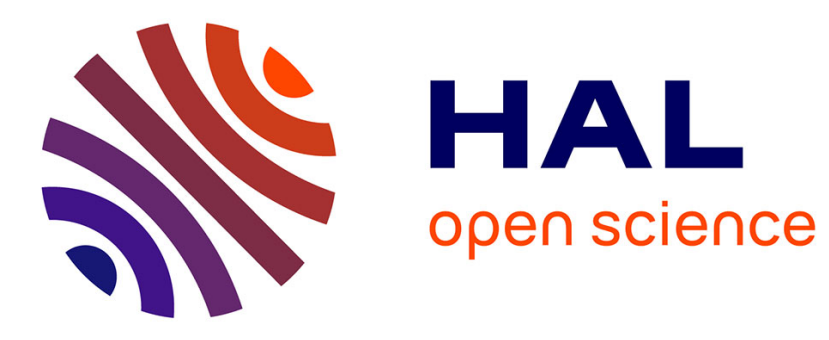

\title{
Failure in rate-independent granular materials as a bifurcation toward a dynamic regime
}

François Nicot, Luc Sibille, Félix Darve

\section{To cite this version:}

François Nicot, Luc Sibille, Félix Darve. Failure in rate-independent granular materials as a bifurcation toward a dynamic regime. International Journal of Plasticity, 2012, 29, pp.136-154. 10.1016/j.ijplas.2011.08.002 . hal-01006913

\section{HAL Id: hal-01006913 https://hal.science/hal-01006913}

Submitted on 4 Sep 2018

HAL is a multi-disciplinary open access archive for the deposit and dissemination of scientific research documents, whether they are published or not. The documents may come from teaching and research institutions in France or abroad, or from public or private research centers.
L'archive ouverte pluridisciplinaire HAL, est destinée au dépôt et à la diffusion de documents scientifiques de niveau recherche, publiés ou non, émanant des établissements d'enseignement et de recherche français ou étrangers, des laboratoires publics ou privés. 


\title{
Failure in rate-independent granular materials as a bifurcation toward a dynamic regime
}

\author{
François Nicot ${ }^{\mathrm{a}, *}$, Luc Sibille ${ }^{\mathrm{b}}$, Félix Darve ${ }^{\mathrm{c}}$ \\ ${ }^{a}$ Cemagref, ETNA - Geomechanics Group, Grenoble, France \\ ${ }^{\mathrm{b}}$ Institut de Recherche en Génie Civil et Mécanique, Université de Nantes, ECN-CNRS, Nantes, France \\ ${ }^{\mathrm{c}}$ Laboratoire Sols Solides Structures, UJF-INPG-CNRS, Grenoble, France
}

\begin{abstract}
This paper investigates the issue of failure in rate-independent granular material specimens under a novel line of reasoning. The mechanism of failure is related to an increase in kinetic energy. Based on energy conservation, expressed in an incremental form, kinetic energy is shown to be related to the so-called second-order work and a boundary integral involving the external loading directed to the system. Thus, the existence of an outburst in kinetic energy is related to a competition between the second-order work and this boundary integral.

Elementary examples are discussed, providing a clear interpretation of the standard laboratory tests (drained, undrained and proportional strain triaxial paths). These theoretical findings are then confirmed based on numerical simulations using a discrete element method. Finally, this approach is assessed in boundary value problems.
\end{abstract}

\section{Introduction}

Generally an effective failure in a material gives rise to bursts of kinetic energy either in a homogeneous sample or in boundary value problems. There is a transition (a bifurcation) from a quasistatic regime (the system admits a unique incremental response over a given incremental loading) toward a dynamical regime (inertial effects govern the response of the system). Effective failure means here that the experimentalist has tried to trespass the limit state reached by an ad hoc additional load or has perturbed the sample or the body at this limit state in an ad hoc way. Effective failure manifests itself by suddenly exponentially growing strains, as observed in laboratory experiments (Darve et al., 2007) and in discrete numerical modelling (Sibille et al., 2008). Moreover, these exponentially growing strains are associated with bursts of kinetic energy, as also observed experimentally and numerically. The purpose of this paper is to explain why effective failure leads to these bursts of kinetic energy in the context of geomaterials, and more specifically of granular materials.

From a theoretical point of view, by taking into account the energy conservation law in continuum mechanics (Mandel, 1966) expressed with a Lagrangian formalism and differentiated with respect to time (Nicot and Darve, 2007; Nicot et al., 2007), it is possible to obtain the kinetic energy as a function of the integrals of the discrete second-order work related to the external and body forces applied minus the integral of the continuous constitutive second-order work in the body. This result is reviewed briefly in the first section of this paper, because it will be used as the basic equation for all further developments.

The second-order work criterion was initially introduced by Hill (1958) as an instability criterion, with the meaning that the material will continue to deform at least in one stress-strain direction without any external input of energy (see also for

\footnotetext{
* Corresponding author.

E-mail address: francois.nicot@cemagref.fr (F. Nicot).
} 
example Petryk (1993); Bigoni (2000) for a review of this issue). This means that a certain failure mode can be induced. Later on, it appears (Valanis, 1989; Bigoni and Hueckel, 1991; Nicot and Darve, 2011) that the second-order work criterion (vanishing value of the determinant of the symmetric part of the elasto-plastic matrix) contains the particular cases of the strain localisation criterion (vanishing value of the determinant of the acoustic matrix) and the plastic limit criterion (vanishing value of the determinant of the elasto-plastic matrix). It was observed (Li and Richmond, 1997; Li and Karr, 2009) that in initially homogenous materials obeying a nonassociated flow rule (the elasto-plastic matrix is non symmetric), plastic deformation is intrinsically unstable. Thus, if flutter instabilities are not considered, the second-order work criterion is the first to be encountered (according to a monotonously increasing loading parameter) (Challamel et al., 2008, 2010) and the other criteria are simply particular cases within the bifurcation domain. The vanishing values of the determinant of the symmetric part of the elasto-plastic matrix corresponds to the internal boundary of the bifurcation domain (Neilsen and Schreyer, 1993; Darve et al., 2004; Sibille et al., 2007; Nicot et al., 2007), while its external boundary is the plastic limit condition. Thus the second-order work criterion can be considered a necessary condition for failure (flutter instabilities excluded) according to these theoretical results. This conclusion was also verified in experiments (Darve et al., 2007) and very carefully checked by direct numerical simulations of failure with a discrete element method (Sibille et al., 2008).

Moreover, it is eventually worth mentioning here that, in grain avalanches, strong correlations have been observed repeatedly in discrete element simulations (Darve et al., 2004) between second-order work and kinetic energy. From a spatial point of view, the area where the second-order work computed on the particle scale ${ }^{1}$ takes negative values coincides with the area of bursts of particle kinetic energy. From a temporal point of view, by considering the changes over time of the total kinetic energy and the total second-order work for all the particles, the peaks of kinetic energy correlate very well with the negative minima of the second-order work. These strong correlations remain unexplained from a theoretical point of view.

Returning to the purpose of this paper and taking into account a convenient expression of kinetic energy, the external second-order work (referred to as the second-order boundary term in the following) exactly balances the positive constitutive second-order work outside the bifurcation domain, thereby maintaining zero kinetic energy. On the contrary, when the bifurcation domain frontier is reached, in one specific loading direction, the constitutive second-order work will vanish (Nicot et al., 2007; Prunier et al., 2009a). If the experimentalist applies an ad hoc additional load, the second-order boundary term takes a strictly positive value, inducing a non-zero kinetic energy and thereby leading to the failure of the sample, or more broadly to the material system considered.

This reasoning is very carefully established in this paper through four successive examples of increasing complexity: first for the classical drained triaxial test where failure occurs on the plastic limit condition for a stress-controlled path, second for the undrained triaxial compression where failure appears strictly inside the plastic limit surface for an axial force control, third for proportional strain loading paths with a generalised failure criterion and an ad hoc mixed control and, finally, in a fourth section, for a foundation and an applied increasing force.

Throughout the paper, only rate-independent materials are considered. Two-order tensors are represented as $\overline{\bar{A}}$, whereas vectors are denoted $\vec{X}$. The summation convention on repeated indices will be employed. For any (one- or two-order) tensor $A,{ }^{t} A$ denotes the transpose tensor. Incremental changes of any variable $y$ are denoted $\delta y$, with $\delta y=\dot{y} \delta t$. When discrete element simulations are considered, incremental changes $\delta y$ are replaced with small finite increments $\Delta y$.

\section{A fundamental equation}

In soil mechanics, many examples of soil specimen collapse have been reported in the literature. Herein, the term "collapse" means that the soil specimen is suddenly no longer able to sustain external forces and experiences large deformation, with strains increasing rapidly over time. This phenomenon is called diffuse failure (Darve et al., 2004), in contrast with localised failure, when the kinematic field presents a chaotic aspect with no visible localisation pattern. In fact, this is a proper bifurcation mode, since there is an abrupt transition from a quasi-static regime towards a dynamical regime. Through a proper formalism, this section investigates how the kinetic energy of a system initially in equilibrium can arise when a certain external incremental loading is applied.

For this purpose, a system made up of a volume $V_{o}$ of a given material, initially in a configuration $C_{o}$ (initial configuration) is considered. $\vec{b}_{0}$ denotes the initial body force density field. After a loading history, the system is in a strained configuration $C$ and occupies a volume $V$, with a body force density field $\vec{b}$, in equilibrium under a prescribed external loading. As a result, an external stress distribution $\vec{r}$ acts on the current boundary $(\Gamma)$ of the material. A part $\left(\Gamma_{\sigma}\right)$ of the current boundary $(\Gamma)$ is subjected to an external stress (the loading is stress controlled on this part of the boundary), whereas the complementary part $\left(\Gamma_{\varepsilon}\right)$ is subjected to kinematic constraints (the loading is strain controlled on this part of the boundary), leading to a reaction external stress.

The instantaneous evolution of the system, in the equilibrium configuration $C$ at time $t$, is governed by the following energy conservation equation that includes dynamical effects:

$$
\delta E_{c}(t)=\int_{\Gamma} r_{i} \delta u_{i} d S+\int_{V} b_{i} \delta u_{i} d V-\int_{V} \sigma_{i j} \frac{\partial\left(\delta u_{i}\right)}{\partial x_{j}} d V
$$

\footnotetext{
${ }^{1}$ The second-order work computed on the particle (or the contact between particles) scale is also referred to as the discrete second-order work, to emphasise that it is computed from local variables, contrary to the macroscopic second-order work computed in the continuum.
} 
where $\delta E_{c}$ represents the system's current change in kinetic energy related to the incremental displacement field $\delta \vec{u}$. $\bar{\sigma}$ is the Cauchy stress tensor. Eq. (1) represents the Euler form of the energy conservation, since all variables are given with respect to the current evolving configuration. In this configuration, any material point is described by the coordinate vector $\vec{x}$. It is worth stressing that the differential notation $\delta Y$ denotes the incremental change in the variable $Y$, so that $\delta Y=\dot{Y} \delta t$. This notation should not be confused with the notation used in the variational principle, where virtual variations in stress or strain fields are considered. Eq. (1) does not correspond to the virtual work theorem but incorporates the real changes in both stress and strain fields over the loading path (here graduated in time).

It is convenient to express the integrals in Eq. (1) with respect to the initial configuration, which yields:

$$
\delta E_{c}(t)=\int_{\Gamma_{o}} f_{i} \delta u_{i} d S_{o}+\int_{V_{o}} b_{o, i} \delta u_{i} d V_{o}-\int_{V_{o}} \Pi_{i j} \frac{\partial\left(\delta u_{i}\right)}{\partial X_{j}} d V_{o}
$$

where $\overline{\bar{\Pi}}$ denotes the Piola-Kirchoff stress tensor of the first type and $\Gamma_{o}$ is the $V_{o}$ boundary. $\overline{\bar{\Pi}}$ (resp. $\vec{f}$ ) is the transformed quantity of $\overline{\bar{\sigma}}$ (resp. $\vec{r}$ ) through the bijection $\vartheta$ mapping the material points from the reference configuration to the current configuration: $\vec{x}=\vartheta(\vec{X})$. This bijective transformation is convenient so as to obtain all integrals given with respect to a fixed domain, that is $\Gamma_{o}$ and $V_{o}$. Thus, the time differentiation of Eq. (2) can be performed in a straightforward manner, without referring to a Reynolds transform. Taking into account Green's formula, differentiating Eq. (2) gives (Nicot et al., 2007).

$$
\delta^{2} E_{c}(t)=\int_{\Gamma_{o}} \delta f_{i} \delta u_{i} d S_{o}+\int_{V_{o}} \delta b_{o, i} \delta u_{i} d V_{o}-\int_{V_{o}} \delta \Pi_{i j} \frac{\partial\left(\delta u_{i}\right)}{\partial X_{j}} d V_{o}
$$

Following Hill's definition (Hill, 1958), $W_{2}=\int_{V_{o}} \delta \Pi_{i j} \delta F_{i j} d V_{o}$ denotes the second-order work of the system, associated with the incremental evolution $\left(\delta \Pi_{i j}, \delta F_{i j}\right)$, where $F_{i j}=\frac{\partial x_{i}}{\partial x_{j}}=\delta_{i j}+\frac{\partial u_{i}}{\partial x_{j}}$ is the general term of the deformation gradient tensor $\overline{\bar{F}}=\overline{\bar{\nabla}} \vartheta$. Both incremental quantities $\delta F_{i j}$ and $\delta \Pi_{i j}$ are related through the constitutive equation. Thus, Eq. (3) also reads:

$$
\delta^{2} E_{c}(t)=\int_{\Gamma_{o}} \delta f_{i} \delta u_{i} d S_{o}+\int_{V_{o}} \delta b_{o, i} \delta u_{i} d V_{o}-W_{2}
$$

In addition, the two-order Taylor expansion of kinetic energy reads:

$$
E_{c}(t+\Delta t)=E_{c}(t)+\Delta t \dot{E}_{c}(t)+\frac{(\Delta t)^{2}}{2} \ddot{E}_{c}(t)+o(\Delta t)^{3}(\forall \Delta t)
$$

Noting that $E_{c}(t)=\frac{1}{2} \int_{V_{o}} \rho_{o}\|\overrightarrow{\vec{u}}\|^{2} d V_{o}$, where $\rho_{o}$ is the density of the material in the initial configuration at point $M(\vec{X})$, since the system is in an equilibrium state at time $t$, then $E_{c}(t)=0$. Furthermore, $\dot{E}_{c}(t)=\int_{V_{o}} \rho_{o} \dot{\vec{u}} \cdot \ddot{\vec{u}} d V_{o}$, and at time $t, \dot{E}_{c}(t)=0$. Eq. (5) therefore reads:

$$
\delta^{2} E_{c}(t)=\frac{2 E_{c}(t+\Delta t)}{(\Delta t)^{2}} \delta t^{2}+o(\Delta t) \delta t^{2}
$$

Thus, Eq. (6) establishes that the kinetic energy of the system at the subsequent time $t+\Delta t$ is a second-order term. Ignoring third-order terms and making $\Delta t \rightarrow \delta t$, then $\delta^{2} E_{c}(t)=2 E_{c}(t+\delta t)$; in combination with Eq. (4), it follows that:

$$
2 E_{c}(t+\delta t)=\int_{\Gamma_{o}} \delta f_{i} \delta u_{i} d S_{o}+\int_{V_{o}} \delta b_{o, i} \delta u_{i} d V_{o}-W_{2}
$$

Eq. (7) is the fundamental equation that relates the kinetic energy of the system to the second-order work. It should be emphasised that Eq. (7) holds true only when the system is in an equilibrium state at time $t$. Eq. (7) shows that the kinetic energy, immediately after an equilibrium state, and in absence of change in body forces, appears as the difference between a boundary term, $B_{2}=\int_{\Gamma_{0}} \delta F_{i} \delta u_{i} d S_{0}$, controlled by the loading path applied to the boundary of the system, and a body constitutive term, $W_{2}=\int_{V_{o}} \delta \Pi_{i j} \frac{\partial\left(\delta u_{i}\right)}{\partial x_{j}} d V_{o}$, that is related to the constitutive behaviour of the material.

Ignoring body forces, Eq. (7) shows that necessarily $\int_{\Gamma_{0}} \delta f_{i} \delta u_{i} d S_{o}-W_{2} \geqslant 0$. In addition, if the incremental evolution is quasi-static, between two equilibrium states, then:

$$
\int_{\Gamma_{0}} \delta f_{i} \delta u_{i} d S_{o}-W_{2}=0
$$

Moreover, as when external stresses are balanced by internal stresses, $\delta f_{i}=\delta \Pi_{i j} N_{j}$ at any point of the boundary $\Gamma_{o}$, where $\vec{N}$ is the normal to the boundary at the point considered. Thus, the following second-order equilibrium equation can be recovered (see Vardoulakis and Sulem, 1995):

$$
\int_{V_{o}} \delta \Pi_{i j} \frac{\partial\left(\delta u_{i}\right)}{\partial X_{j}} d V_{o}=\int_{\Gamma_{o}} \delta \Pi_{i j} N_{j} \delta u_{i} d S_{o}
$$

In this paper, the case where both external and internal stresses are unbalanced is investigated, that is:

$$
\int_{\Gamma_{o}} \delta f_{i} \delta u_{i} d S_{o}>W_{2}
$$


According to Eq. (7), this leads to an outburst in kinetic energy $\left(E_{c}(t+\delta t)>0\right)$, in absence of body forces. The occurrence of an outburst in kinetic energy is therefore related to a conflict between the loading prescribed to the boundary of the system and its bearing capacity dictated by its constitutive behaviour. The influence of body forces will be discussed in a forthcoming paper. This is a nontrivial situation, of great importance in view of developing a proper mechanical framework to describe the occurrence of mass-driven phenomena, such as landslides.

The paper is organised as follows. First, the case of the material point scale (Representative Volume Element) is considered, and standard loading paths are examined from a theoretical point of view. Then this investigation is corroborated by numerical simulations run with a discrete element method. Finally, these developments are generalised to boundary value problems, by considering a shallow foundation.

Throughout the following sections, the context of large strains is considered, and the investigation is carried out using a Lagrangian formalism involving both the Piola-Kirchoff tensor $\overline{\bar{\Pi}}$ and the deformation gradient tensor $\overline{\bar{F}}$.

\section{The laboratory specimen scale}

The above-mentioned theoretical framework is now considered on the laboratory specimen scale. Investigating this elementary scale can be useful in, for example, laboratory tests and the interpretation of the derived experimental results, where parallelepiped-like specimens subjected, on each wall, to a prescribed force or displacement directing both stress and strain fields can be studied.

\subsection{Local formulation}

Let a parallelepiped specimen be considered, with three sides ( $i=4,5,6)$ fixed. Sides ' $i$ ' and ' $i+3$ ' $(i=1,2,3)$ are assumed to remain parallel. The initial volume is $V_{o}$, the initial area of each side ' $i$ ' $(i=1,2,3)$ of normal $\vec{v}_{i}$ is denoted $A_{i}$ and the initial length of each edge is denoted $L_{i}$. Index ' 1 ' refers to the axial direction (major principal direction), whereas indices ' 2 ' and ' 3 ' refer to the two lateral directions perpendicular to the axial direction (Fig. 1). When a static condition is assigned to a side ' $i$ ', it is convenient to introduce the resultant external force $f_{i}$ acting on this side. This force is assigned to be normal to the face considered. The uniform external stress vector distribution $s_{i}$ acting on side ' $i$ ' and related to $f_{i}$, is also introduced: $s_{i}=\frac{f_{i}}{A_{i}}$. The displacement of each side ' $i$ ', along the direction $\vec{v}_{i}$, is denoted $U_{i}=\vec{u} \cdot \vec{v}_{i}$. No tangential displacement is assumed to take place. When a kinematic condition is assigned to a side ' $i$ ', the resultant external force $f_{i}$ (or the stress vector distribution $s_{i}$ ) acting on this side corresponds to the external loading that must be applied to ensure the prescribed displacement $U_{i}$. In this context:

$$
\langle\overline{\bar{F}}\rangle=\left[\begin{array}{ccc}
\left\langle F_{11}\right\rangle & 0 & 0 \\
0 & \left\langle F_{22}\right\rangle & 0 \\
0 & 0 & \left\langle F_{33}\right\rangle
\end{array}\right] \text { and }\langle\overline{\bar{\Pi}}\rangle=\left[\begin{array}{ccc}
\left\langle\Pi_{11}\right\rangle & 0 & 0 \\
0 & \left\langle\Pi_{22}\right\rangle & 0 \\
0 & 0 & \left\langle\Pi_{33}\right\rangle
\end{array}\right]
$$

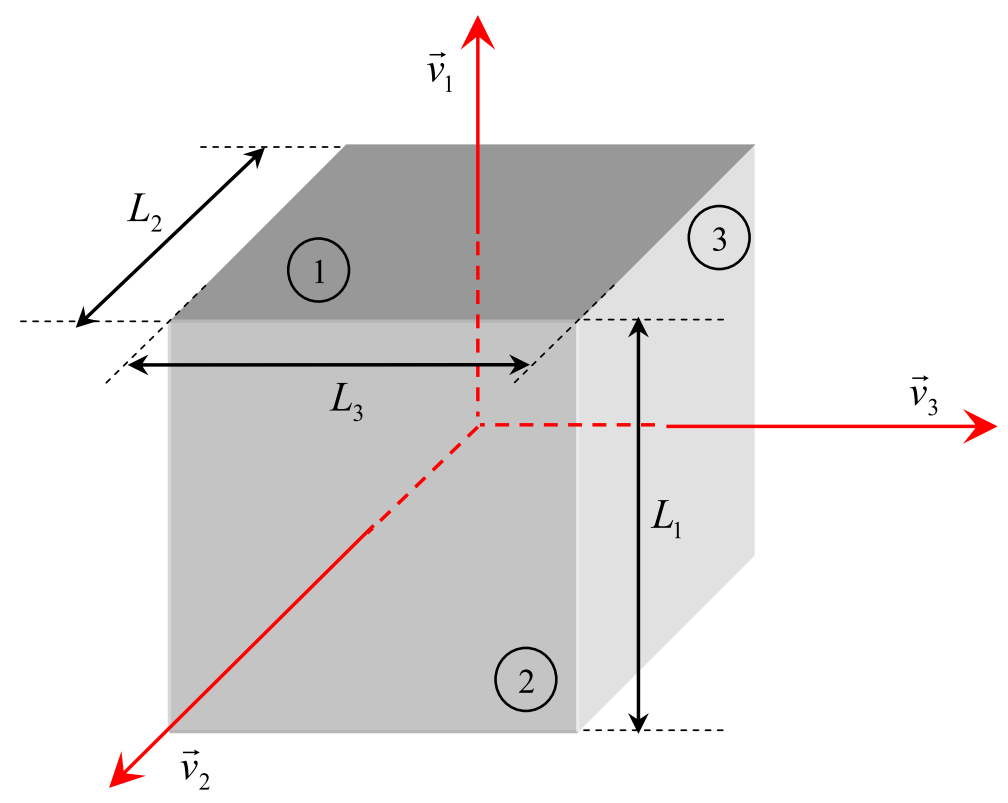

Fig. 1. Parallelepiped specimen and definition of the axes. 
where $\langle Y\rangle=1 / V_{o} \int_{V_{o}} Y d V$ denotes the mean value of the variable $Y$ over the whole volume $V_{o}$.

As $\left\langle F_{i i}\right\rangle=\frac{1}{V_{o}} \int_{V_{o}}\left(1+\frac{\partial u_{i}}{\partial X_{i}}\right) d V$, by virtue of the Green formula, the following holds:

$$
\left\langle F_{i i}\right\rangle=\frac{1}{V_{o}} \int_{\partial V_{o}} u_{i} v_{i} d S=1+\frac{A_{i}}{V_{o}} U_{i} \text { (with no summation on repeated index ' } i \text { ') }
$$

Moreover, the macro-homogeneity assumption makes it possible to invoke the fundamental Hill identity, stating that $\left\langle\delta \Pi_{i j} \delta F_{i j}\right\rangle=\left\langle\delta \Pi_{i j}\right\rangle\left\langle\delta F_{i j}\right\rangle$. In these conditions, the second-order reads:

$$
W_{2}=\int_{V_{o}} \delta \Pi_{i j} \delta F_{i j} d V_{o}=V_{o}\left\langle\delta \Pi_{i j} \delta F_{i j}\right\rangle=V_{o}\left\langle\delta \Pi_{i j}\right\rangle\left\langle\delta F_{i j}\right\rangle
$$

From above, Eq. (7) is expressed as:

$$
2 E_{c}(t+\Delta t)=A_{1} \delta s_{1} \delta U_{1}+A_{2} \delta s_{2} \delta U_{2}+A_{3} \delta s_{3} \delta U_{3}-V_{o}\left(\left\langle\delta \Pi_{11}\right\rangle\left\langle\delta F_{11}\right\rangle+\left\langle\delta \Pi_{22}\right\rangle\left\langle\delta F_{22}\right\rangle+\left\langle\delta \Pi_{33}\right\rangle\left\langle\delta F_{33}\right\rangle\right)
$$

Noting that the initial volume of the specimen is given by:

$$
V_{o}=L_{1} A_{1}=L_{2} A_{2}=L_{3} A_{3}=L_{1} L_{2} L_{3}
$$

then $A_{i}=V_{o} / L_{i}$. As $\delta U_{i}=L_{i}\left\langle\delta F_{i i}\right\rangle$ (with no summation on repeated index ' $i$ '), Eq. (14) can be expressed as:

$$
2 E_{c}(t+\Delta t)=V_{o} \sum_{i=1}^{3}\left(\delta s_{i}-\left\langle\delta \Pi_{i i}\right\rangle\right)\left\langle\delta F_{i i}\right\rangle
$$

In the sequel, for purposes of convenience, terms $\left\langle F_{i i}\right\rangle$ (resp. $\left.\left\langle\delta F_{i i}\right\rangle\right)$ and $\left\langle\Pi_{i i}\right\rangle$ (resp. $\left.\left\langle\delta \Pi_{i i}\right\rangle\right)$ are denoted $F_{i}\left(\right.$ resp. $\left.\left\langle\delta F_{i}\right\rangle\right)$ and $\Pi_{i}$ (resp. $\delta \Pi_{i}$ ).

It is essential to distinguish the forces (or stress vectors) acting on the boundary of the specimen, with stress acting within the specimen. The constitutive response of the specimen is characterised by a relation between incremental strain $\delta F_{i}$ and stress $\delta \Pi_{i}$ components. However, the only information that can be obtained is recorded on the boundary, and therefore involves $s_{i}$ and $U_{i}$. During a quasistatic evolution of the specimen, along successive equilibrium states, the internal stress tensor within the specimen is balanced with the external stress vector, so that for each side ' $i$ ' $\Pi_{i}=s_{i}$. Thus, the constitutive response of the specimen can be investigated in that case from the measurable variables $s_{i}$ and $U_{i}$ (or $F_{i}=U_{i} / L_{i}+1$ ). This is exactly what is done during laboratory tests. This is sound until the specimen fails: if inertial effects take place, the external stress is not balanced by the internal stress, and a heterogeneous strain field may develop within the specimen.

\subsection{The drained triaxial test in axisymmetric conditions}

In this test, an axisymmetric loading is applied by prescribing a constant axial displacement rate $\delta U_{1}=L_{1} \delta F_{1}$, together with a constant lateral pressure $\left(s_{2}=s_{3}\right)$. For dense specimens, the curve giving the evolution of $s_{1}$ versus $U_{1}$ passes through a peak, and then tends towards a plateau, as seen in Fig. 2. $\hat{s}_{1}$ denotes the value of $s_{1}$ at the peak. During this quasistatic

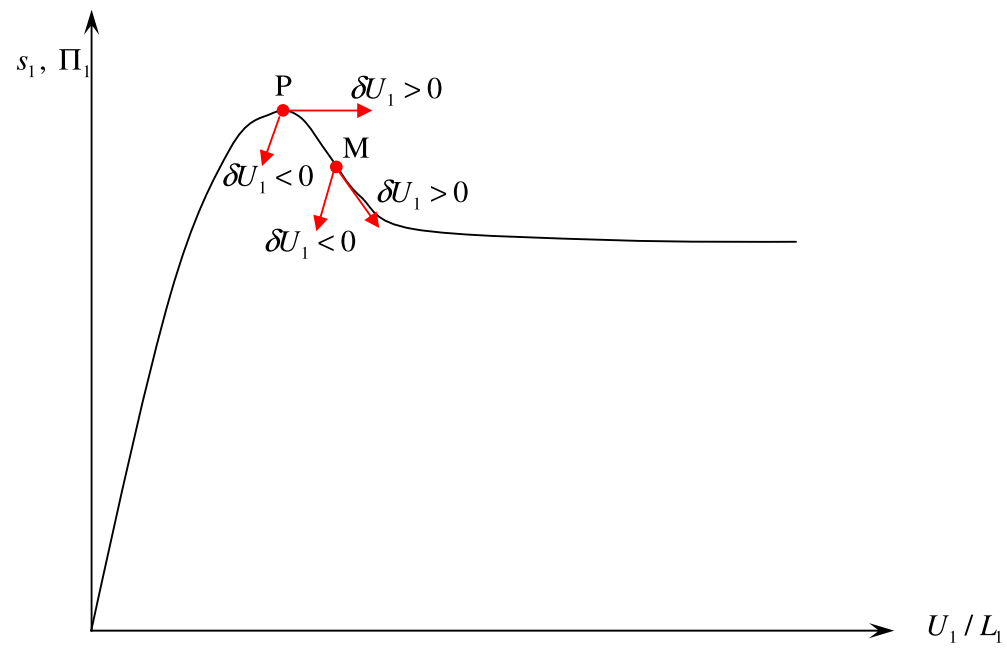

Fig. 2. Drained triaxial test. Incremental stress direction during loading or unloading, at the axial stress peak (point $\mathrm{P}$ ) and during the softening regime (point M). 
evolution, using this loading mode, $\Pi_{1}=s_{1}$. The second-order work for the specimen of volume $V$ reads $W_{2}=V_{o} \delta \Pi_{1} \delta F_{1}$ along this loading path.

Let us focus on the axial stress peak (point P). At this point, a stress loading control is now adopted, so that an external axial stress $\hat{s}_{1}$ is applied to the specimen, the lateral stress $\left(s_{2}=s_{3}\right)$ being kept constant. The equilibrium state is unchanged, and the internal stress within the specimen remains in equilibrium with the external stress. Let an additional infinitesimal axial loading $\delta s_{1}$ be applied to the specimen at time $t$. As shown in Appendix A, $\delta U_{1}>0$. Thus, the additional axial loading $\delta s_{1}$ also corresponds to an increase in the incremental axial displacement $\delta U_{1}$. It is essential to note that the incremental internal stress $\delta \bar{\Pi}$ within the specimen, due to the application of $\delta s_{1}=\delta f_{1} / A_{1}$ at time $t$, is the same as that which would exist if the incremental axial displacement $\delta U_{1}$ was indeed imposed. Thus, $\delta \Pi_{3}=0$ and according to the constitutive response of the specimen shown in Fig. 2, $\delta \Pi_{1}=0$ at the peak. The second-order work is therefore nil. Moreover, since the lateral confinement is unchanged, $\delta s_{3}=0$. As a consequence,

$$
2 E_{c}(t+\Delta t)=V_{o} \delta s_{1} \delta F_{1}-W_{2}=A_{1} \delta s_{1} \delta U_{1}=\delta f_{1} \delta U_{1}>0
$$

At the peak, under the effect of an additional axial loading, the specimen's kinetic energy increases from zero to a strictly positive value $\delta f_{1} \delta U_{1}$ over the infinitesimal time interval [ $t, t+\delta t[$. The failure mechanism of the specimen is initiated. In fact, the internal axial stress $\sigma_{1}$ within the specimen is no longer able to balance the external axial stress $s_{1}$ : $s_{1}$ is kept constant (equal to $\hat{s}_{1}+\delta s_{1}$ ), whereas $\Pi_{1}$ follows a constitutive path and decreases from $\hat{s}_{1}$ along the descending branch. The unbalanced axial stress is responsible for the dynamical response of the specimen, characterised in Eq. (17) by a strictly positive value of $E_{c}(t+\delta t)$. Note that an infinitesimal external axial load is sufficient to provoke the failure of the specimen.

It is worth noting that Eq. (17), which holds true only from an equilibrium state (time $t$ ), describes the incremental evolution of the system according to a second-order analysis. Eq. (17) therefore holds over time intervals $[t, t+\delta t[$ for small values of $\Delta t$. For larger values of $\Delta t$, the dynamical evolution of the system directs likely nonhomogeneous strain and stress fields, leading to the definitive failure of the specimen. This failure evolution, beyond its initiating stage, cannot be described by Eq. (17), nor more generally by Eq. (7). This conclusion will be confirmed by discrete element simulations in Section 3.5 .

The same reasoning can be applied at any point after the peak. Along the descending branch, whatever the sign of $\delta U_{1}$, $\delta s_{1}<0$. Thus, at point M (Fig. 2), the second-order work is strictly negative if $\delta U_{1}>0$ (loading) and strictly positive if $\delta U_{1}<0$ (unloading).

Now let an infinitesimal axial loading $\delta s_{1}$ be applied to the specimen at point M (Fig. 2). Necessarily $\delta U_{1}>0$ (see Appendix A). The second-order work is therefore strictly negative. As a consequence,

$$
2 E_{c}(t+\delta t)=A_{1} \delta s_{1} \delta U_{1}-W_{2}=A_{1}\left(\delta s_{1}-\delta \Pi_{1}\right) \delta U_{1}>0
$$

The failure mechanism of the specimen is therefore initiated, and Eq. (18) clearly shows that this failure is related to the unbalance between external $\left(\delta s_{1}\right)$ and internal $\left(\delta \Pi_{1}\right)$ axial stresses acting on the axial side ' 1 ' of the specimen.

\subsection{The undrained triaxial test in axisymmetric conditions}

The undrained triaxial test consists in imposing a constant axial displacement rate $\left(\delta U_{1}=L_{1} \delta F_{1}\right)$, while the volume of the specimen is kept constant $\left(\delta F_{1} /\left(1+F_{1}\right)+2 \delta F_{3} /\left(1+F_{3}\right)=0\right)$. As reported in an abundant literature (Castro, 1969; Lade and Pradel, 1990; Lade, 1992; Biarez and Hicher, 1994; Chu et al., 2003), the experimental curve giving the evolution of the deviatoric stress $q_{s}=s_{1}-s_{3}$ versus the axial displacement passes through a peak for sufficiently loose specimens (Fig. 3 ).

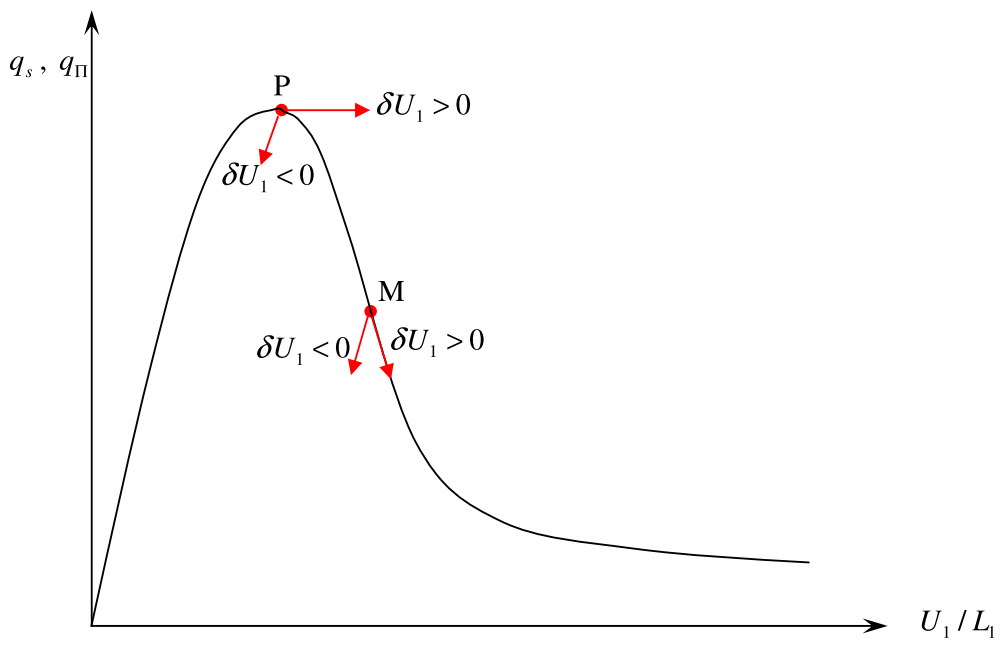

Fig. 3. Undrained triaxial test. Incremental stress direction during loading or unloading, at the axial stress peak (point P) and during the softening regime (point M). 
The same reasoning as that considered in the previous section can be applied at the peak $\mathrm{P}$ or at a given point $\mathrm{M}$ along the descending branch. An updated Lagrangian configuration will be considered in the subsequent analysis carried out at any of these two points, so that the isochoric condition simplifies into $\delta F_{1}+2 \delta F_{3}=0$.

Using this strain control loading mode $\left(\delta U_{1}>0\right.$ and $\left.\delta V=0\right)$, the response of the specimen follows a quasistatic evolution: $\Pi_{i}=s_{i}$, and the curve in Fig. 3 can be given indifferently in terms of $q_{s}=s_{1}-s_{3}$ or $q_{\Pi}=\Pi_{1}-\Pi_{3}$. Thus, the second-order work for the specimen of volume $V_{o}$ reads:

$$
W_{2}=V_{o}\left(\left(\delta \Pi_{1}-\delta \Pi_{3}\right) \delta F_{1}+\delta \Pi_{3}\left(\delta F_{1}+2 \delta F_{3}\right)\right)=V_{o} \delta q_{\Pi} \delta F_{1}
$$

Let us focus on the deviatoric stress peak (point $\mathrm{P}$ ). At this equilibrium point, the internal stress directs both an axial stress $\hat{s}_{1}$ and a lateral stress $\hat{s}_{3}$ to the boundary of the specimen. Imagine that the axial loading is henceforth stress controlled, so that a deviatoric load $\hat{q}_{s}=\hat{s}_{1}-\hat{s}_{3}$ is applied. No change in the volume of the specimen is assigned. Let an infinitesimal deviatoric stress loading $\delta q_{s}>0\left(\delta q_{s}=\delta s_{1}-\delta s_{3}=\delta f_{1} / A_{1}\right)$ be applied to the specimen (Fig. 3$)$. The loading considered directs a strictly positive incremental evolution of the axial displacement $\delta U_{1}$ (see Appendix B). $\delta U_{1}=L_{1} \delta F_{1}>0$. The second-order work reads:

$$
W_{2}=V_{o} \delta q_{\Pi} \delta F_{1}=A_{1} \delta q_{\Pi} \delta U_{1}
$$

It should be noted that the internal stress within the specimen due to the application of $\delta q_{s}$ is the same as that which would exist if the incremental axial displacement $\delta U_{1}$ was effectively imposed. Thus, according to the constitutive response of the specimen shown in Fig. $2, \delta q_{\Pi}=0$ at the peak. The second-order work is therefore nil: $W_{2}=0$.

Moreover,

$$
\delta s_{i} \delta F_{i}=\delta q_{s} \delta F_{1}+\left(\delta F_{1}+2 \delta F_{3}\right) \delta s_{3}=\delta q_{s} \delta F_{1}
$$

As a consequence,

$$
2 E_{c}(t+\delta t)=V_{o} \delta q_{s} \delta F_{1}-W_{2}>0
$$

As for the drained triaxial test, if an infinitesimal loading is applied at the deviatoric stress peak, the specimen is expected to fail. The external force $\left(A_{1} \delta q_{s}\right)$ cannot be balanced by the internal stress. An unbalanced stress is generated, responsible for the dynamical response of the specimen. This is exactly what is observed experimentally (Castro, 1969; Lade and Pradel, 1990; Lade, 1992; Chu et al., 2003; Darve et al., 2007) and numerically when using a discrete element method (Sibille et al., 2008; Darve et al., 2007). If the loading is strain controlled $\left(\delta U_{1}>0\right.$ and $\left.\delta V=0\right)$, and for sufficiently loose specimens, a static liquefaction is observed: both the effective mean pressure and deviatoric stress vanish without a dynamical effect. In contrast, if the test is stress controlled $\left(\delta q_{s}>0\right.$ and $\left.\delta V=0\right)$, then the specimen abruptly fails at (or just after) the deviatoric stress peak.

\subsection{The proportional strain loading path}

The investigation is now generalised to the proportional strain loading path, where a constant axial displacement rate $\dot{U}_{1}=L_{1} \dot{F}_{1}$ is imposed, while the ratio between both axial and lateral strain rates ( $\dot{F}_{1}$ and $\left.\dot{F}_{3}\right)$ is kept constant. In incremental form,

$$
\delta F_{1}+2 \lambda \delta F_{3}=0
$$

As far as axisymmetric conditions hold, this monotonic incremental loading is general. The direction of the loading can be chosen within the incremental strain path, according to the value of $\lambda, \delta U_{1}$ being the loading parameter.

For both drained and undrained triaxial loading paths, the occurrence of a failure was dictated by the existence of a peak for a stress variable: the axial stress $\Pi_{1}$ for the drained triaxial test, the deviatoric stress $q_{\Pi}$ for the undrained triaxial test. In the context of a proportional strain path, the general stress variable $\Pi_{1}+\mu \Pi_{3}$ can be considered. As constitutive equations read in axisymmetric conditions (to transpose the metrics, $\delta \Pi_{1}$ and $\sqrt{2} \delta \Pi_{3}$ are related to $\delta F_{1}$ and $\sqrt{2} \delta F_{3}$ ).

$$
\begin{aligned}
& \delta \Pi_{1}=K_{11} \delta F_{1}+2 K_{12} \delta F_{3} \\
& \sqrt{2} \delta \Pi_{3}=\sqrt{2} K_{21} \delta F_{1}+\sqrt{2}\left(K_{22}+K_{23}\right) \delta F_{3}
\end{aligned}
$$

where $\overline{\bar{K}}$ is the tangent constitutive matrix relating $\delta \vec{F}$ and $\delta \vec{\Pi}$.

It follows that:

$$
\delta \Pi_{1}+\mu \delta \Pi_{3}=\left(K_{11}+\mu K_{21}\right) \delta F_{1}+\left(2 K_{12}+\mu\left(K_{22}+K_{23}\right)\right) \delta F_{3}
$$

As $\delta F_{1}+2 \lambda \delta F_{3}=0$, Eq. (25) yields:

$$
\delta \Pi_{1}+\mu \delta \Pi_{3}=\left(K_{11}+\mu K_{21}-\frac{1}{\lambda} K_{12}-\frac{\mu}{\lambda} \frac{K_{22}+K_{23}}{2}\right) \delta F_{1}
$$


which can be rewritten as:

$$
\delta \Pi_{1}+\mu \delta \Pi_{3}=\underline{\underline{X}} \underline{\underline{K Y}} \delta F_{1}
$$

with $\underline{\underline{K}}=\left[\begin{array}{cc}K_{11} & \sqrt{2} K_{12} \\ \sqrt{2} K_{21} & K_{22}+K_{23}\end{array}\right], \underline{X}^{t}=\left[\begin{array}{ll}1 & \frac{\mu}{\sqrt{2}}\end{array}\right]$ and $\underline{Y}=\left[\begin{array}{c}1 \\ -\frac{1}{\sqrt{2} \lambda}\end{array}\right]$.

Noting that $\underline{\underline{K}}=\underline{\underline{K^{s}}}+\frac{K_{21}-K_{12}}{\sqrt{2}}\left[\begin{array}{cc}0 & -1 \\ 1 & 0\end{array}\right]$ with $\underline{\underline{\underline{K}}}=\left[\begin{array}{cc}K_{11} & \frac{K_{21}-K_{12}}{\sqrt{2}} \\ \frac{K_{21}-K_{12}}{\sqrt{2}} & K_{22}+K_{23}\end{array}\right]$ are the symmetric part of $\underline{\underline{K}}$, Eq. (27) finally reads:

$$
\delta \Pi_{1}+\mu \delta \Pi_{3}=\underline{X}^{t} \underline{\underline{K}} \underline{Y} \delta F_{1}+\left(\mu+\frac{1}{\lambda}\right) \frac{K_{21}-K_{12}}{2} \delta F_{1}
$$

This expression simplifies when both the stress variable $\Pi_{1}+\mu \Pi_{3}$ and the strain variable $F_{1}+2 \lambda F_{3}$ verify the energy conjugation condition: couples $(\alpha, \beta)$ exist such that:

$$
\Pi_{1} F_{1}+2 \Pi_{3} F_{3}=\alpha\left(\Pi_{1}+\mu \Pi_{3}\right)+\beta\left(F_{1}+2 \lambda F_{3}\right)
$$

$\alpha$ being a linear combination of $F_{1}$ and $F_{3}$, and $\beta$ being a linear combination of $\Pi_{1}$ and $\Pi_{3}$.

Eq. (29) requires that:

$$
\mu+\frac{1}{\lambda}=0
$$

The stress variable thus reads $\Pi_{1}-\frac{1}{\lambda} \Pi_{3}$. For $\lambda=1$ (isochoric conditions), the deviatoric term $q_{s}=s_{1}-s_{3}$ is recovered.

Taking Eq. (30) into account, Eq. (28) simplifies to:

$$
\delta \Pi_{1}-\frac{1}{\lambda} \delta \Pi_{3}=\underline{X}^{t} \underline{K}^{s} \underline{X} \delta F_{1}
$$

with $\underline{X}=\left[\begin{array}{c}1 \\ \frac{-1}{\sqrt{2} \lambda}\end{array}\right]$.

Moreover, the second-order work $W_{2}(\lambda)$ associated with the incremental strain direction $\underline{X}$ is related to the matrix $\underline{\underline{K}}^{s}(\mathrm{Ni}$ cot et al., 2009) as follows:

$$
W_{2}(\lambda)=V_{0} \underline{X}^{t} \underline{K}^{s} \underline{X}\left(\delta F_{1}\right)^{2}
$$

As a consequence, Eqs. (30) and (31) become:

$$
\delta \Pi_{1}-\frac{1}{\lambda} \delta \Pi_{3}=\frac{W_{2}(\lambda)}{V_{o} \delta F_{1}}
$$

Thus, the stress variable $\Pi_{1}-\frac{1}{\lambda} \Pi_{3}$ admits a peak if and only if the second-order work is vanishing along the direction $\underline{X}=\left[\begin{array}{c}1 \\ \frac{-1}{\sqrt{2}}\end{array}\right]$ corresponding to the condition $\delta F_{1}+2 \lambda \delta F_{3}=0$, where $F_{1}+2 \lambda F_{3}$ is the conjugate strain variable.

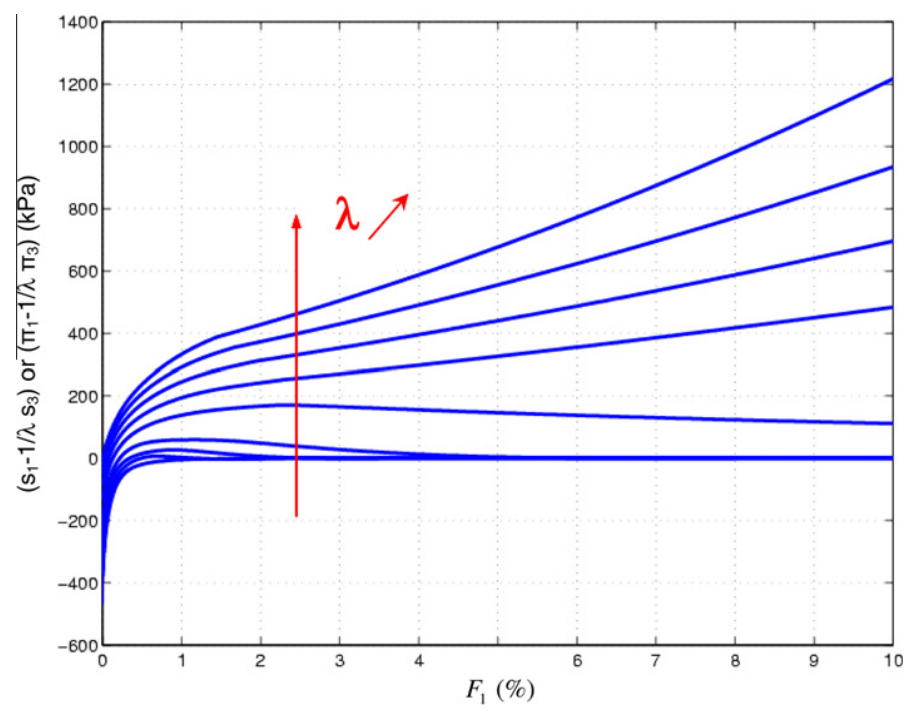

Fig. 4. Proportional strain triaxial loading paths. From Darve et al. (2004). 
Fig. 4 illustrates how $s_{1}-\frac{1}{\lambda} s_{3}$ evolves in terms of $F_{1}$, by considering a piece-wise incrementally linear constitutive relation (Darve et al., 2004). Interestingly, it can be observed that the curve passes through a peak for sufficiently small values of $\lambda$. In what follows, such a $\lambda$-value is considered.

As the response of the specimen follows a quasistatic evolution along the proportional strain loading path, then $\Pi_{i}=s_{i}$. Thus, as $\delta F_{1}+2 \lambda \delta F_{3}=0$, the second-order work for the specimen of volume $V_{o}$ reads:

$$
W_{2}=V_{o}\left(\left(\delta \Pi_{1}-\frac{1}{\lambda} \delta \Pi_{3}\right) \delta F_{1}+\frac{1}{\lambda} \delta \Pi_{3}\left(\delta F_{1}+2 \lambda \delta F_{3}\right)\right)=V_{o}\left(\delta \Pi_{1}-\frac{1}{\lambda} \delta \Pi_{3}\right) \delta F_{1}
$$

Likewise:

$$
\delta s_{1} \delta F+2 \delta s_{3} \delta F_{3}=\left(\delta s_{1}-\frac{1}{\lambda} \delta s_{3}\right) \delta F_{1}+\frac{1}{\lambda} \delta s_{3}\left(\delta F_{1}+2 \lambda \delta F_{3}\right)=\left(\delta s_{1}-\frac{1}{\lambda} \delta s_{3}\right) \delta F_{1}
$$

Thus, Eq. (16) reads:

$$
2 E_{c}(t+\delta t)=V_{o}\left(\delta q_{s}^{\lambda}-\delta q_{\Pi}^{\lambda}\right) \delta F_{1}
$$

with $q_{s}^{\lambda}=s_{1}-\frac{1}{\lambda} s_{3}$ and $q_{\Pi}^{\lambda}=\Pi_{1}-\frac{1}{\lambda} \Pi_{3}$.

At any point along the descending branch, both internal lateral stress $\widehat{\Pi}_{3}$ and axial stress $\widehat{\Pi}_{1}$ exist within the specimen, directing external stresses $\hat{s}_{3}$ and $\hat{s}_{1}, \hat{s}_{1}=\widehat{\Pi}_{1}$ and $\hat{s}_{3}=\widehat{\Pi}_{3}$. Imagine that a mixed control mode is adopted, so that the external stress $\hat{s}_{1}-\frac{1}{2} \hat{s}_{3}$ is imposed, together with the kinematic condition $\delta F_{1}+2 \lambda \delta F_{3}=0$. Let an infinitesimal stress loading $\delta q_{s}^{\lambda}>0$ be applied to the specimen. Thus, $\delta F_{1}>0$ (see Appendix B). It should be noted that the incremental internal stress within the specimen due to the application of $\delta s_{1}$ is the same as that which would exist if the incremental axial strain $\delta F_{1}$ was imposed. Thus, according to the constitutive response of the specimen shown in Fig. $4, \delta q_{\Pi}^{\lambda}<0$. As a consequence, according to Eq. (36), $E_{c}(t+\delta t)>0$.

Finally, the same conclusions as those derived in the previous sections hold: the constitutive response of the specimen makes the internal stress decrease. The internal stress inside the specimen is therefore not able to balance the external stress applied, and a dynamical regime takes place, leading to the failure of the specimen associated with a transition from a quasistatic regime towards a dynamical regime.

\subsection{Numerical inspection using a discrete element method}

In this section, inspection is carried out from numerical experiments based on the discrete element method DEM (Cundall and Strack, 1979) and conducted with the YADE code (Kozicki and Donzé, 2008). In the discrete element method, each particle constituting the granular assembly is explicitly described. Inertial terms are taken into account for each particle and its position is updated at each time step by an explicit time integration of dynamic fundamental equations. Consequently, the response of a granular assembly simulated by DEM is a dynamic response. However, as for real laboratory tests, a quasi-static response (where the influence of inertial terms is negligible) can be assessed for a sufficiently low loading rate. Moreover, given that inertial terms are taken into account, the DEM can be used as a tool to investigate the development of the effective failure (leading to a dynamic response) from an equilibrium state when the system, made up of particles, is controlled in such a way that effective failure can occur. In this section, DEM is mainly used within this latter framework in order to compare the increase in kinetic energy just after the occurrence of effective failure, with the balance between the loading prescribed at the boundary of the system and the bearing capacity of this system related to its constitutive behaviour.

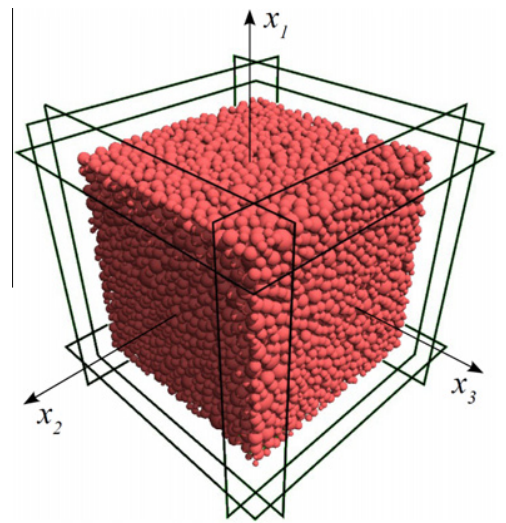

(a)

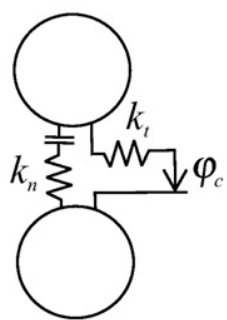

(b)

Fig. 5. The particle assembly enclosed with six rigid and frictionless walls (a) the inter-particle contact law (b). 
The three-dimensional model developed is presented in Fig. 5(a) and consists of a cubical assembly of 10,000 particles enclosed within six rigid and frictionless walls. A representative elementary volume can be estimated by checking the reproducibility of the response to a given loading simulated from granular assemblies initially generated with different random particle positions. An example of a test of reproducibility is presented in Appendix C for 10,000 particles in 3D. This number of particles is a satisfactory compromise between the computation cost and result reproducibility, and Plassiard et al. (2009) showed that increasing the number of particles beyond 10,000 does not affect the results significantly.

Particles are spheres with a continuous size distribution describing the grading of Fontainebleau sand. The walls are square plates with a finite width, length and thickness; they can be subjected to Newton's second law according to their mass, as any particle constituting the numerical sample.

In the normal contact direction, the inter-particle contact behaviour (Fig. 5(b)) is modelled by an elastic force-displacement relation characterised by the stiffness $k_{n}\left(k_{n} / D_{s}=250 \mathrm{MPa}\right.$, where $D_{s}$ is the sphere diameter). No tensile force is allowed. In directions belonging to the tangent contact plane, the force-displacement relation is elastic-perfectly plastic and characterised by the stiffness $k_{t}\left(k_{t} / k_{n}=0.5\right)$ and the friction angle $\varphi_{c}=35^{\circ}$.

Since there is no tangential force in wall-grain contacts, the principal stress and strain directions coincide with the normal directions to the walls. To prescribe a given loading program, each principal stress and strain component can be imposed on the boundaries of the particle assembly by acting on the walls. In a given principal direction $i$, the average strain value $F_{i}$ is directly imposed by adjusting the wall displacements (in this case, walls do not follow the dynamic fundamental equations). For a stress control, walls are considered dynamic objects (like particles), they can translate along their normal direction according to Newton's second law, but their rotation is locked. An external force $f_{i}^{\text {ext }}$ is applied to the walls, and the walls move until reaching equilibrium. Then an external stress distribution $s_{i}=f_{i}^{\text {ext }} / A_{i}$ develops on the boundary of the sample. This type of stress control was adopted to simulate the application of a real force control resulting from the application of additional external masses. Moreover, contrary to the case of a closed-loop control (Sibille et al., 2009), it directly gives access to the value of the external stress $s_{i}$ (if the walls' inertia is small enough). To prevent too long a delay in the application of $s_{i}$, due to inertial effects (that would transform the force control into a kind of strain control), a low value has been attributed to the mass of each wall, corresponding to $1 \%$ of the total mass of the particle assembly.
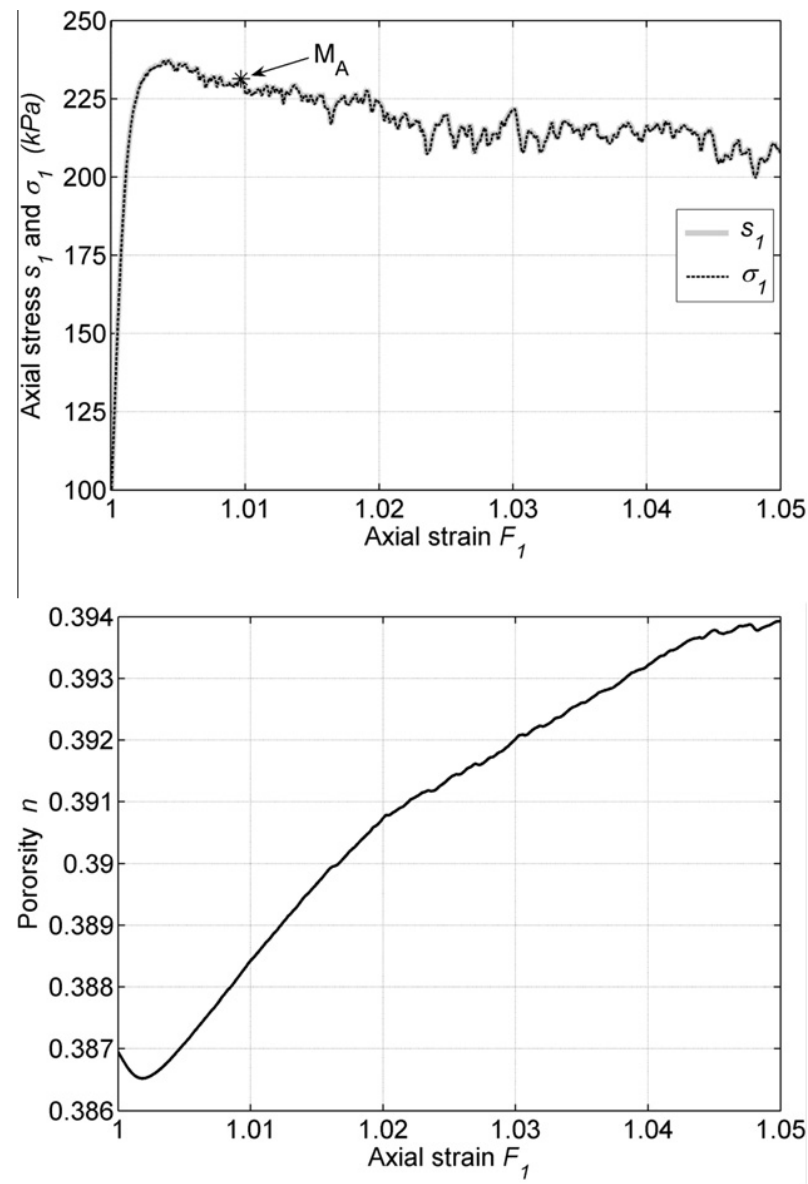

Fig. 6. Triaxial drained compression on the dense granular assembly, the internal axial stress $\sigma_{1}$ and the axial stress computed at the boundary of the sample $s_{1}$ are here equal. 
In the following simulations, only both the average stress and average strain states are assessed. The average strain state is determined from wall positions. For the sake of simplicity, the average internal Cauchy stress state is computed with the Love-Weber formula (see for instance Love, 1927; Weber, 1966). Thus an Eulerian expression for the stress tensor is used, instead of a Lagrangian expression. Even though the theoretical approach was developed in a Lagrangian formalism, the main results inferred in the previous sections will be checked from an Eulerian approximation of the stress terms:

$$
\sigma_{i j}=\frac{1}{V} \sum_{\text {contacts }} r_{i}^{c} l_{j}^{c}
$$

where $\overrightarrow{r^{c}}$ represents the inter-particle contact force and $\vec{l}$ the branch vector joining the centres of the two particles involved in the contact. In the same way, an Eulerian approximation of the external stress distribution is considered. When the sample is strain controlled in direction $i$, the external stress distribution $s_{i}$ is computed as $s_{i}=f_{i} / A_{i}$ where $A_{i}$ is the current area of side ' $i$ ', and $f_{i}$ is the reaction force of the sample on the wall, equal to the sum of all sphere-wall contact forces. When the sample is force controlled, $s_{i}$ can also be computed as $s_{i}=f_{i}^{\text {ext }} / A_{i}$, where $f_{i}^{e x t}$ is the external force applied to the wall ' $i$ '.

First, the case of the drained triaxial compression on a dense sample is investigated. The sample is subjected to an isotropic compression up to a confining pressure of $100 \mathrm{kPa}$. At this stage, the porosity is $n=0.387$ and the coordination number is $z=4.53$. Then the sample is compressed by imposing a constant axial strain rate $\dot{F}_{1}=0.01 \mathrm{~s}^{-1}$ while the radial stresses $s_{2}$ and $s_{3}$ are kept constant, equal to $100 \mathrm{kPa}$. For this simulation where a quasi-static response is expected, the inertial effects can be estimated through the inertial number $I$ which describes the ratio of inertial to pressure forces (da Cruz et al., 2005). It is defined in 3D for a particle of diameter $D_{s}$ and mass $m$ by $I=\dot{F}_{1} \frac{m}{D_{s} P}$, where $P$ is the pressure confinement applied to the granular assembly. A value of $I=10^{-2}$ corresponds to the very limit of a quasi-static loading (da Cruz et al., 2005) and a lower value should be respected to simulate a quasi-static loading with the DEM. In the present case, the maximum value (for the largest particle) is $I=1.5 \times 10^{-4}$. A series of simulations at different loading rates can also give an indication of the range of loading rates to apply in order to avoid the inertial effect; this approach is illustrated in Appendix D.

The sample's response to this drained triaxial compression is analysed in terms of axial stress, $s_{1}$ or $\Pi_{1}$, and porosity, as shown in Fig. 6. During this quasi-static evolution, it is shown that $s_{1}$ and $\Pi_{1}$ remain very close.

Let us now consider the state $M_{A}$ after the peak of $s_{1}$ (Fig. 6). In this state, the sample control is switched to a full stress control defined by $s_{1}=\sigma_{1}^{M_{A}}=231.3 \mathrm{kPa}$ and $s_{2}=s_{3}=100 \mathrm{kPa}$, until the sample stabilises under this imposed external stress state. Then a small external axial stress increment $\Delta s_{1}=2 \mathrm{kPa}$ is imposed, representing less than $1 \%$ of $\sigma_{1}^{M_{A}}$ (the lateral confining is kept constant: $s_{2}=s_{3}=100 \mathrm{kPa}$ ). $\Delta s_{1}$ is not imposed instantaneously, but gradually within 400 numerical time steps, in order to limit the development and the propagation of elastic waves. In this case, where a quasi-static response of the sample is not imposed, the strain rate is not imposed by the operator but is actually a response of the sample to the small additional stress increment $\Delta s_{1}$. However, over the range of time considered in the following figures, the inertial number $I$ does not exceed $9.10^{-4}$ and inertial forces remain quite low with respect to pressure forces.

Changes in stress increments are presented in Fig. 7. The internal stress $\sigma_{1}$ first increases because the sample has slightly strengthened during the stabilisation stage at the initial stress state $\sigma_{1}^{M_{A}}$. However, $\sigma_{1}$ rapidly decreases, moving away from the applied external stress $s_{1}$. Since $\sigma_{1}$ is no longer able to balance $s_{1}$, the kinetic energy strongly increases, as shown in Fig. 8 .

$\left[W_{2}\right]_{\Delta t},\left[V \Delta s_{i} \Delta F_{i}\right]_{\Delta t}$ and $\left[\left(V \Delta s_{i} \Delta F_{i}-W_{2}\right) / 2\right]_{\Delta t}$ over the time period $\Delta t$, are given in Fig. 8. The negative sign of $W_{2}$ is related to a negative value of $\Delta \sigma_{1}$, whereas $\Delta F_{1}$ is positive, while $V\left(\Delta s_{1} \Delta F_{1}\right)$ is positive since $\Delta s_{1}>0$. As expected from Eq. (16), good agreement is obtained between $\left[E_{c}\right]_{\Delta t}$ and $\left[\left(V \Delta s_{i} \Delta F_{i}-W_{2}\right) / 2\right]_{\Delta t}$ for $\Delta t<0.05 \mathrm{~s}$. The computation of the last expression is based on the values of $\Delta F_{i}$ and $\Delta \sigma_{i}$ over a given time period $\Delta t$, whereas their time series are strongly nonlinear and nonmonotonous. Consequently, the term $\left[\left(V \Delta s_{i} \Delta F_{i}-W_{2}\right) / 2\right]_{\Delta t}$ corresponds to a secant value over the time period $\Delta t$, whereas

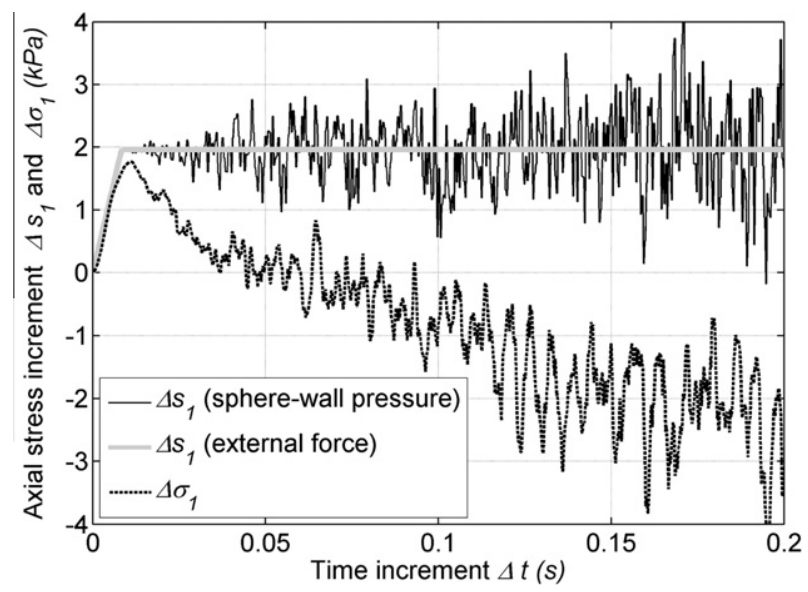

Fig. 7. Time versus the stress increments resulting from the application of $\Delta s_{1}=2 \mathrm{kPa}$ from the state $M_{A}$ defined in Fig. 6 . 

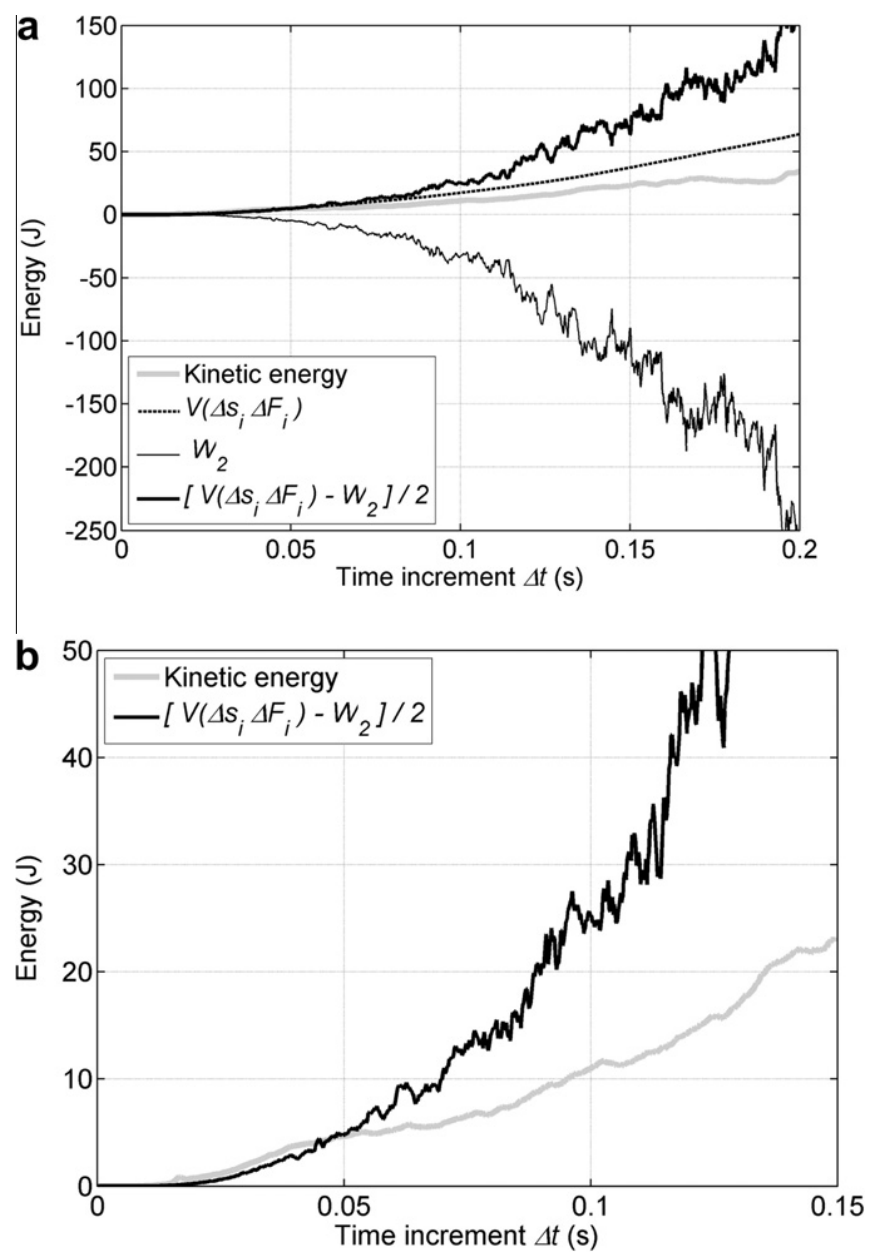

Fig. 8. Time series of the kinetic energy and terms $\left[W_{2}\right]_{\Delta t},\left[V \Delta s_{i} \Delta F_{i}\right]_{\Delta t}$, and $\left[\left(V \Delta s_{i} \Delta F_{i}-W_{2}\right) / 2\right]_{\Delta t}$ during and after the application of $\Delta s_{1}=2 \mathrm{kPa}$ from the state $M_{A}(\mathrm{a})$. Focus on shorter time increments (b).

the kinetic energy integrates the whole history of $F_{i}$ and $\sigma_{i}$ over the time increment $\Delta t$, explaining why the numerical results depart from Eq. (16) when $\Delta t$ increases. Moreover, Eq. (16) was established for an infinitesimally small time increment $\delta t$ from an initial equilibrium state. However, with direct numerical simulations only finite time increments $\Delta t$ are applicable. It is shown here that Eq. (16) holds true for time increments that are not too long (here less than $0.05 \mathrm{~s}$ ) and fails for larger $\Delta t$, since Eq. (6) is no longer valid.

The case of the undrained (isochoric) triaxial compression with a loose granular assembly is now considered. As for the drained triaxial compression, the sample is first subjected to an isotropic compression up to a confining pressure of $100 \mathrm{kPa}$ (where $n=0.403$ and $z=3.55$ ). Then the isochoric condition $\Delta V / V=\Delta F_{1}+\Delta F_{2}+\Delta F_{3}=0$ is imposed by adjusting, at each numerical time step, the displacements of walls along the normal directions 2 and 3 , such as $\Delta F_{2}=\Delta F_{3}=-\Delta F_{1} / 2$. Together with the isochoric constraint, the sample is compressed at the constant axial strain rate $\dot{F}_{1}=0.01 \mathrm{~s}^{-1}$. As shown in Fig. 9, after a maximum of the stress deviator $q_{s}$, stresses totally vanish (the sample is liquefying). However, one can observe that this sample is at the limit of liquefaction under the isochoric condition. After the maximum of $q_{s}$, the sample temporally regains strength, as shown by the local peak of $q_{s}$. This type of sample was purposely chosen to make stabilisation easier at the state $M_{B}$ after the maximum of $q_{s}$, as indicated in Fig. 9 . At this state, the sample control is switched to a stress control by imposing a deviatoric stress $q_{s}=q_{\sigma}^{M_{B}}=43 \mathrm{kPa}$ (while the isochoric condition is still applied; see Sibille et al., 2009, for the implementation of the mixed stress-strain control mode), until the sample stabilises. If the assembly of spheres is very loose, this stabilisation of the sample at a prescribed axial stress after the maximum of $q_{s}$ is almost impossible to achieve.

After stabilising the sample at the state $M_{B}$, an additional external deviatoric stress $\Delta q_{s}=1.6 \mathrm{kPa}$ (corresponding to $3.7 \%$ of $q_{\sigma}^{M_{B}}$ ) is gradually imposed within 240 numerical time steps (the isochoric condition is maintained). It is worth emphasising that the strain rate is not imposed so that an effective failure can possibly develop with a burst of kinetic energy. Changes in stress increments are given in Fig. 10.

As shown in the case of drained conditions, the internal deviatoric stress $q_{\sigma}$ first increases (possibly due to a kind of residual strength acquired during the stabilisation phase), then decreases to depart from the deviatoric stress $q_{s}$ applied to the 

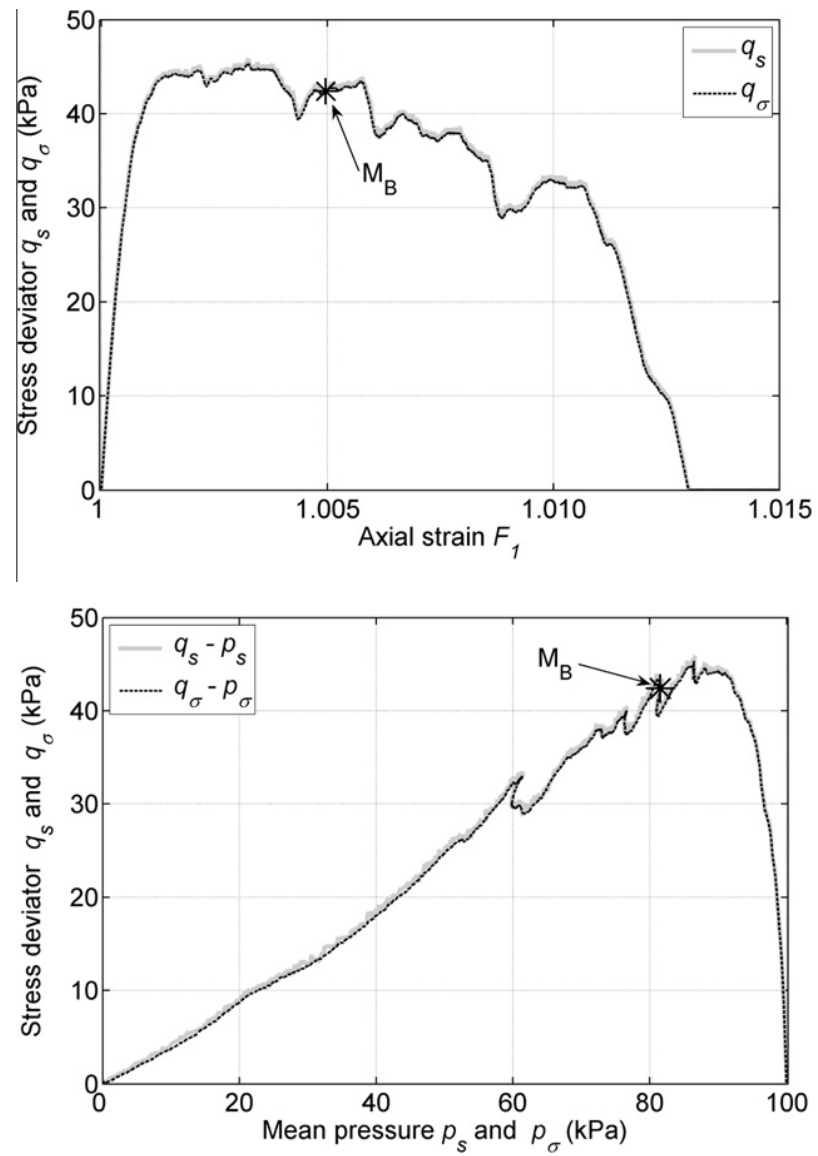

Fig. 9. Undrained (isochoric) triaxial compression of a loose particle assembly, $q_{s}$ and $p_{s}$ are computed from the stress state at the boundary of the sample, $q_{\sigma}$ and $p_{\sigma}$ are computed from the internal stress state.

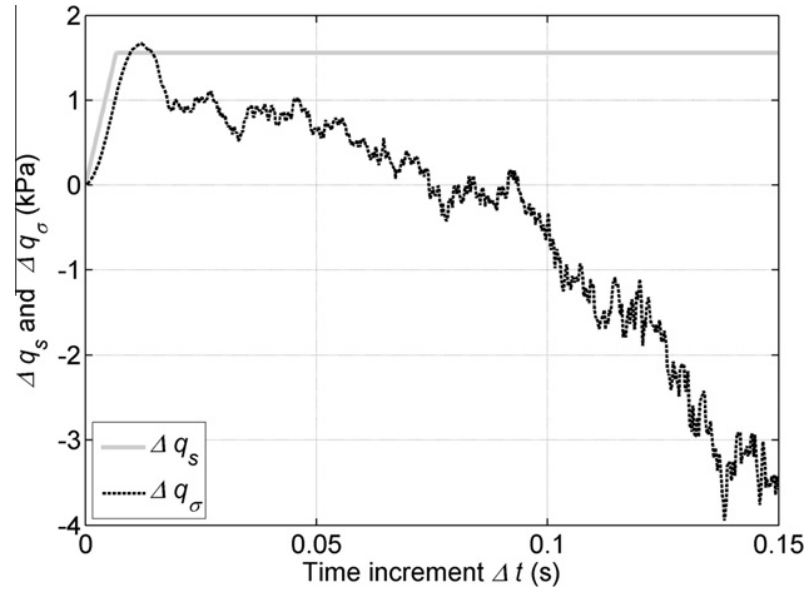

Fig. 10. Time versus the stress increments resulting from the application of $\Delta q_{s}=1.6 \mathrm{kPa}$ from the state $M_{B}$ defined in Fig. 9 .

boundary of the sample. Due to this unbalanced state, the kinetic energy rapidly increases, as shown in Fig. 11, and good agreement is found between $\left[E_{c}\right]_{\Delta t}$ and $\left[\left(V\left(\Delta s_{i} \Delta F_{i}\right)-W_{2}\right) / 2\right]_{\Delta t}$ up to $\Delta t$ values of about $0.08 \mathrm{~s}$ (similar to the limit $\Delta t$ value observed in drained conditions).

It is worth noting that failure develops as $W_{2}$ takes decreasing negative values (Fig. 11). The boundary term of Eq. (16) remains greater than $W_{2}$, leading to a positive value of $E_{c}$ at the end of each time increment $\Delta t$. 


\section{Extension to the boundary value problem}

The case of a boundary value problem leading to a bifurcation state is examined in this section, with the settlement of a soil body under a shallow foundation. The problem is assumed to be two-dimensional and is modelled as described in Fig. 12. A rectangular domain of soil is considered; a part $J K$ of length $L$ of the upper side is subjected to a controlled downward ver-
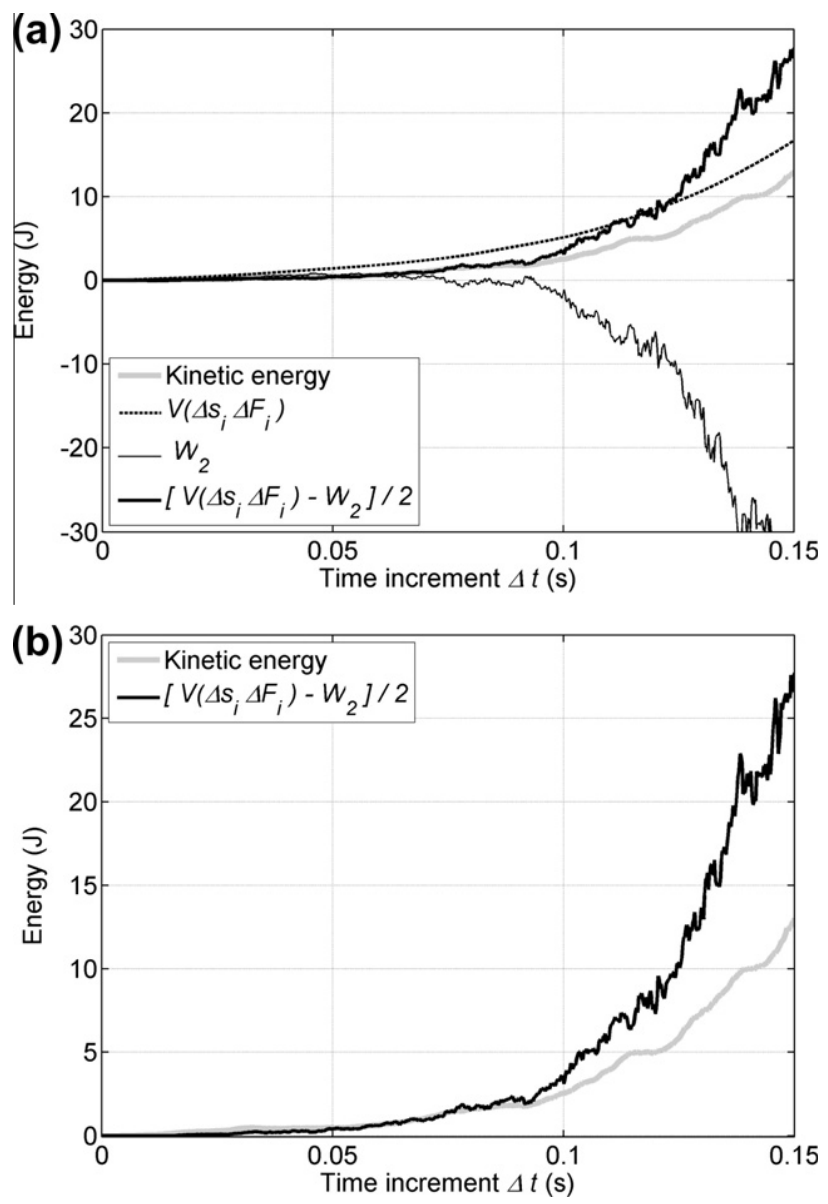

Fig. 11. Time series of the kinetic energy and terms $\left[W_{2}\right]_{\Delta t},\left[V \Delta s_{i} \Delta F_{i}\right]_{\Delta t}$, and $\left[\left(V \Delta s_{i} \Delta F_{i}-W_{2}\right) / 2\right]_{\Delta t}$ during and after the application of $\Delta q_{s}=1.6 \mathrm{kPa}$ from the state $M_{B}(\mathrm{a})$. Focus on shorter time increments (b).

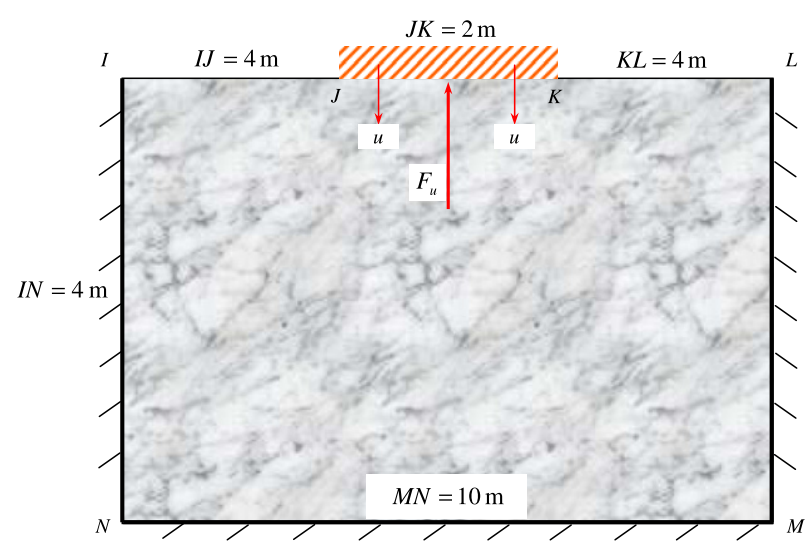

Fig. 12. Simulation of the settlement under a shallow foundation. 
tical displacement denoted $u$, whereas the adjoining parts (IJ and $K L)$ are free. The other three sides $(L M, M N$ and $I N)$ are restricted from undergoing displacement. In contrast to what was done in Section 3, the mechanical state within the soil body is not homogeneous.

The reaction force applied by the soil to the foundation is denoted $F_{u}$. This force evolves continuously with the vertical displacement $u$. Omitting body forces (negligible here with respect to the external applied force), the boundary term of Eq. (7) is written:

$$
B_{2}=L \delta u \delta F_{u}
$$

Khoa (2005) has simulated this problem by means of a finite element method (LAGAMINE software, developed at Liege University) using the PLASOL elastic-plastic model for soil (Barnichon, 1998). A comprehensive review of this method can be found in Prunier et al. (2009b), Lignon et al. (2009). It has been shown that the curve $F_{u}(u)$ increases until a peak $\widehat{F}_{u}$ is reached, and then decreases (Fig. 13). The kinematic control ensures that no failure occurs. Internal stresses inside the soil body are in balance with the external loading exerted on the boundary. Eq. (8) therefore yields:

$$
W_{2}=B_{2}=L \delta u \delta F_{u}
$$

Thus, given a strictly positive incremental displacement $\delta u$, the second-order work linked to the external applied incremental loading is nil exactly at the peak and is negative along the descending branch (Fig. 14).

Imagine that at the peak, the loading is stress controlled (a force $\widehat{F}_{u}$ is imposed on the foundation) and that a subsequent incremental force $\delta F_{u}>0$ is imposed at time $t$. Following the same reasoning as previously, it can be shown that the incremental displacement $\delta u$ is strictly positive. Exactly at the peak, this positive incremental displacement $\delta u$ directs a nil value of the second-order work.

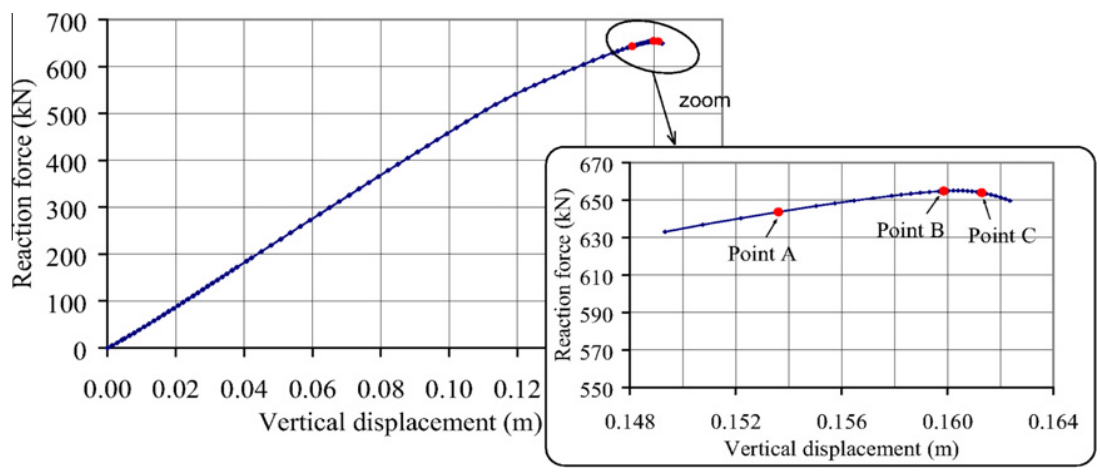

Fig. 13. Reaction force applied to the foundation.

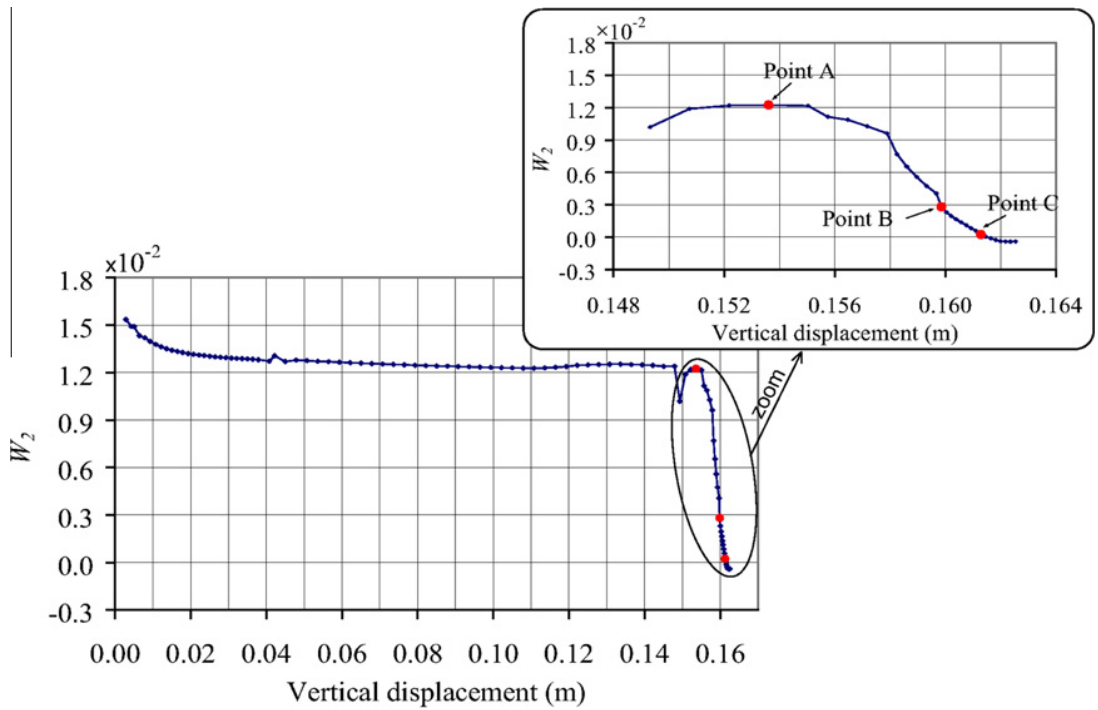

Fig. 14. Normalised second-order work. 
As we have:

$$
2 E_{c}(t+\delta t)=V_{o} \delta u \delta F_{u}-W_{2}=V_{o} \delta u \delta F
$$

where $V_{o}$ is the initial volume of the soil body, the kinetic energy is strictly positive immediately after time $t$. The soil fails under the foundation. This was ascertained from a numerical simulation based on a finite element method using the PLASOL elasto-plastic model for soil. LAGAMINE software considers static balance equations, omitting inertial terms. If an additional (even infinitesimal) load is applied to the foundation immediately after the peak $\widehat{F}_{u}$, then the software is no longer able to converge towards a solution. This numerical divergence is in fact related to a transition from a static to a dynamical regime that cannot be simulated by the software. This transition is the consequence of a sudden increase in kinetic energy.

\section{Concluding remarks}

This paper has investigated the issue of failure in specimens made up of granular, rate-independent materials. Basically, failure of a material is described as the incapacity of this material to sustain an external loading. When a granular material fails, in absence of localisation patterns, a chaotic kinematic field develops, with an abrupt increase in the strain rate. Starting from this physical evidence, the approach developed in this paper is based on energy conservation, leading to a basic equation that introduces the second-order work. More exactly, the increase in the kinetic energy from an equilibrium state, under an incremental loading, is shown to be equal to the difference between a boundary term involving the external loading parameters and the second-order work. The purpose of this paper was to investigate how the boundary term can be greater than the second-order work, leading to an increase in kinetic energy. The mechanical reason behind this is based on a distinction between the internal stress within the material, and forces or stresses applied to the boundary of the system. When a stress limit state is reached, a certain stress component passes through a maximum and then may decrease. This feature corresponds to a vanishing or a negative value of the second-order work. At this point, or along the descending branch, if a certain additional external loading is applied, the system fails, sharply increasing the strain rates. The internal stress is no longer able to balance the external stress, leading to a dynamic response of the specimen.

This theoretical framework was explored along a variety of loading paths, including drained and undrained triaxial paths, and proportional strain triaxial paths as well. For the drained triaxial test, the stress limit state corresponds to the Mohr-Coulomb plastic limit. For the undrained triaxial test, the stress limit state coincides with a maximum of the deviatoric stress, occurring for loose materials well before the Mohr-Coulomb plastic limit is reached (Lade and Pradel, 1990; Lade, 1992). This is an extension of the previous case, generalising the notion of the stress limit state. Going ahead, for denser materials, a stress limit state is observed (before the Mohr-Coulomb plastic limit is reached) over proportional strain triaxial paths, for certain ratios between both axial and lateral incremental strains. The theoretical approach properly shows why these (generalised) stress limit states lead to an increase in kinetic energy and therefore to the failure of the materials. This is in line with the investigation of Valanis (1985) or Bažant and Cedolin (1991), querying the uniqueness of the solution in the softening regime. This paper extends the case of softening to that of generalised limit state with a mixed strain and stress loading control.

These theoretical conclusions were ascertained from numerical simulations based on a discrete element method. If an (even very small) additional loading is applied after a stress limit state is reached, then the numerical specimen is shown to collapse, with an abrupt increase in axial strain rates.

The last section was devoted to an extension towards boundary value problems. A shallow foundation was considered, and the resulting force applied to the foundation when applying a settlement was simulated using a finite element method. The same conclusions hold true. After a limit state is reached, if an additional loading is applied, the soil body under the foundation collapses. Large strains develop, and the convergence of the software fails.

Finally, this approach developed on physical bases suggests an interpretation of the failure mechanisms in solid materials. The most important feature is that failure is related to a transition from a static (equilibrium) regime towards a dynamical regime. As suggested in many papers from the pioneering contribution of Hill (1958), the second-order work is shown to play a basic role. Many applications are expected in engineering sciences, such as soil failure in soil mechanics. In particular, the influence of body forces has to be accounted for to describe the occurrence of mass-driven phenomena, such as landslides. The context of structure mechanics should also be considered. Some results have already been obtained in this direction (Challamel et al., 2010).

The micromechanical understanding of failure in structured granular soils can be regarded as a natural continuation of the current work. Some attempts were already made along this line (Kuhn and Chang, 2006; Nicot and Darve, 2006; Wan et al., 2010; Tordesillas and Muthuswamy, 2009; Tordesillas et al., 2010; Steinhauser et al., 2009; Tsutsumi and Kaneko, 2008). For this purpose, using micromechanically-based models (see for example Chang and Hicher, 2005; La Ragione et al., 2008; Zhu et al., 2010) could be a powerful approach to penetrate down into the microstructural scale.

\section{Acknowledgments}

The French Federative Research Structure VOR (Natural Hazards and Structure Vulnerability) is gratefully acknowledged by the authors. The European project LESSLOSS, and the French national (ANR) SIGMA, STABROCK and Snow-White projects also supported this research. 


\section{Appendix A}

The axial stress peak (point P), in Fig. 2, is the subject of analysis. If $\delta U_{1}>0$ then $\delta \Pi_{1}=0$. If an unloading is performed, $\delta U_{1}<0$ and $\delta \Pi_{1}<0$. Thus, at this peak, the second-order work is zero if $\delta U_{1}>0$ (loading) and strictly positive if $\delta U_{1}<0$ (unloading).

Now let an infinitesimal axial loading $\delta s_{1}$ be applied to the specimen at time $t$. This loading directs an incremental evolution of the axial displacement $\delta U_{1}$. Whatever the sign of $\delta U_{1}$, the incremental stress field is homogeneous: $\delta \Pi_{3}=\delta s_{3}=0$, and $\delta \Pi_{1}$ is given by the constitutive response of the system, as shown in Fig. 2. In what follows, we query the sign of $\delta U_{1}$.

If $\delta U_{1}<0$, the second-order work is strictly positive and we have, according to Eq. (16):

$$
2 E_{c}(t+\delta t)=A_{1} \delta s_{1} \delta U_{1}-W_{2}<A_{1} \delta s_{1} \delta U_{1}
$$

As $\delta s_{1}>0$ and $\delta U_{1}<0$, it follows that $E_{c}(t+\delta t)<0$, which is not possible. Thus, necessarily $\delta U_{1}>0$.

\section{Appendix B}

The deviatoric stress peak (point P), in Fig. 3, is now examined. If $\delta U_{1}>0$ then $\delta q_{\Pi}=0$. If an unloading is performed, $\delta U_{1}<0$ and $\delta q_{\Pi}<0$. Thus, at this peak, the second-order work is zero if $\delta U_{1}>0$ (loading) and strictly positive if $\delta U_{1}<0$ (unloading).

Now let an infinitesimal positive deviatoric stress loading $\delta q_{s}$ be applied to the specimen, together with the isochoric condition $\delta F_{1}+2 \delta F_{3}=0$ (Fig. 3). This loading directs an incremental evolution of the axial displacement $\delta U_{1}$. How does $\delta U_{1}$ evolve? If $\delta U_{1}<0$, the second-order work is strictly positive and we have, according to Eq. (16):

$$
2 E_{c}(t+\delta t)=V\left(\delta s_{1} \delta F_{1}+2 \delta s_{3} \delta F_{3}\right)-W_{2}<V\left(\delta s_{1} \delta F_{1}+2 \delta s_{3} \delta F_{3}\right)
$$

As $\delta s_{1} \delta F_{1}+2 \delta s_{3} \delta F_{3}=\left(\delta s_{1}-\delta s_{3}\right) \delta F_{1}+\delta s_{3}\left(\delta F_{1}+2 \delta F_{3}\right)=\delta q_{s} \delta \tilde{\varepsilon}_{1}$, with $\delta q_{s}>0$ and $\delta F_{1}=\delta U_{1} / L_{1}<0$, it follows that $E_{c}(t+\delta t)<0$, which is not possible. Thus, necessarily $\delta U_{1}>0$.

This result can be generalised to proportional strain loading paths $\left(\delta F_{1}+2 \lambda \delta F_{3}=0\right)$. If $\delta U_{1}<0$, Eq. (A.2) holds, and:

$$
\delta s_{1} \delta F_{1}+2 \delta s_{3} \delta F_{3}=\left(\delta s_{1}-\frac{1}{\lambda} \delta s_{3}\right) \delta F_{1}+\frac{1}{\lambda} \delta s_{3}\left(\delta F_{1}+2 \lambda \delta F_{3} t\right)=\left(\delta s_{1}-\frac{1}{\lambda} \delta s_{3}\right) \delta F_{1}
$$

with $\delta s_{1}-\frac{1}{\lambda} \delta s_{3}<0$ and $\delta F_{1}<0$; it follows that $E_{c}(t+\delta t)<0$, which is not possible. Thus, necessarily $\delta U_{1}>0$.

\section{Appendix C}

For simulations of homogenous tests with the discrete element method, granular assemblies involving a sufficiently large number of particles (as for real laboratory tests) should be considered such that the size of the greatest heterogeneity (here comprising the largest particle) is small with respect to the size of the granular assembly. If this scaling is respected, the response of the granular assembly to a homogenous loading is independent of its local heterogeneities. This latter point has been investigated in 3D by performing classical triaxial compressions (samples are compressed in the axial direction while the radial stress is kept constant) on ten cubical granular assemblies composed of 10,000 particles. Each granular assembly is initially generated from an algorithm positioning particles randomly. Hence all granular assemblies have identical macroscopic properties (porosity, particle size distribution, etc.) but different local heterogeneities. The results presented in Fig. 15 show that fairly good reproducibility is assessed with 10,000 particles.
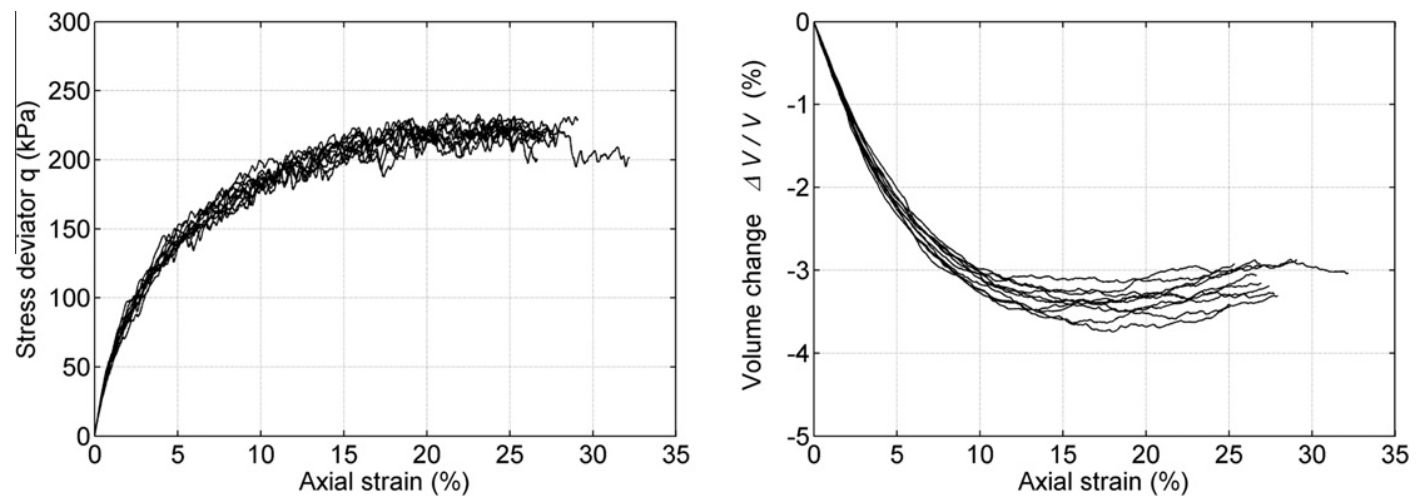

Fig. 15. Simulation of a drained triaxial compression under a confinement of $200 \mathrm{kPa}$ on ten loose granular assemblies composed of 10,000 randomly generated particles. 

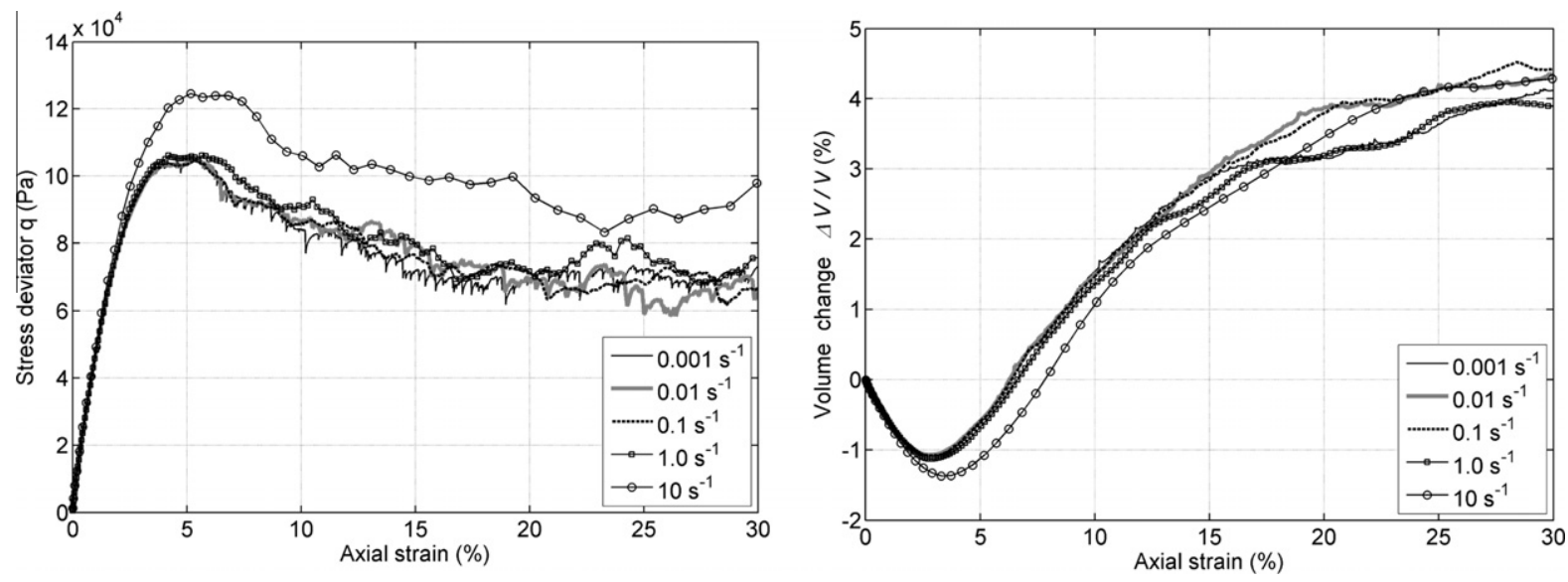

Fig. 16. Simulations of drained triaxial compressions under a confinement of $50 \mathrm{kPa}$ at different axial strain rates $\left(0.001 ; 0.01 ; 0.1 ; 1.0\right.$ and $\left.10 \mathrm{~s}^{-1}\right)$.

\section{Appendix D}

The influence of the loading strain rate and inertial terms is presented in Fig. 16, where a drained triaxial compression is simulated by DEM with a dense granular assembly by imposing the axial compression at different strain rates (all other parameters are kept constant). For sufficiently low strain rate values (here from and below $0.1 \mathrm{~s}^{-1}$ ) the results are independent of the loading rate and thus of the inertial terms. The simulated response can be considered quasi-static for these low strain rate values. For the largest strain rates $\left(1 \mathrm{~s}^{-1}\right.$ and $\left.10 \mathrm{~s}^{-1}\right)$, the quasi-static shear strength (maximum of the stress deviator $q$ ) of the granular assembly is overestimated due to non-negligible inertial forces with respect to pressure forces. Similarly, the maximum contractancy (the minimum volume change) is also overestimated.

\section{References}

Barnichon, J.D., 1998. Finite Element Modeling in Structural and Petroleum Geology. Ph.D. Thesis, Université de Liège.

Bažant, Z., Cedolin, L., 1991. Stability of Structures: Elastic, Inelastic, Fracture, and Damage Theories. Dover Edition Publ., Inc., New-York.

Biarez, J., Hicher, P.Y., 1994. Elementary Mechanics of Soil Behaviour, Saturated Remoulded Soils. Balkema.

Bigoni, D., Hueckel, T., 1991. Uniqueness and localization. I: Associative and non-associative elastoplasticity. Int. J. Solids Struct. 28 (2), $197-213$.

Bigoni, D., 2000. Bifurcation and instability of non-associative elastoplastic solids. In: Petryk, H. (Ed.), Material Instabilities in Elastic and Plastic Solids, CISM Courses and Lecturers, vol. 414. Springer-Verlag, pp. 1-52.

Castro, G., 1969. Liquefaction of sands. Harvard Soil Mechanics Series, vol. 81. Harvard University.

Challamel, N., Nicot, F., Lerbet, J., Darve, F., 2008. On the stability of non-conservative elastic systems under mixed perturbations. EJECE 13 (3), $347-367$.

Challamel, N., Nicot, F., Lerbet, J., Darve, F., 2010. A theoretical link between second-order work and stability under kinematics constraints. Eng. Struct. 32, 3086-3092.

Chang, C.S., Hicher, P.Y., 2005. An elasto-plastic model for granular materials with microstructural consideration. Int. J. Solids Struct. 42 (14), $4258-4277$.

Chu, J., Leroueil, S., Leong, W.K., 2003. Unstable behavior of sand and its implication for slope instability. Can. Geotech. J. 40, 873-885.

Cundall, P.A., Strack, O.D.L., 1979. A discrete numerical model for granular assemblies. Géotechnique 29, 47-65.

da Cruz, F., Emam, S., Prochnow, M., Roux, J.N., Chevoir, F., 2005. Rheophysics of dense granular materials: discrete simulation of plane shear flows. Phys. Rev. E $72(2), 021309$.

Darve, F., Servant, G., Laouafa, F., Khoa, H.D.V., 2004. Failure in geomaterials, continuous and discrete analyses. Comput. Methods Appl. Mech. Eng. 193, 3057-3085.

Darve, F., Sibille, L., Daouadji, A., Nicot, F., 2007. Bifurcations in granular media, macro- and micro-mechanics. Compte Rend. Acad. Sci. - Méc. 335, $496-515$.

Hill, R., 1958. A general theory of uniqueness and stability in elastic-plastic solids. J. Mech. Phys. Solids 6, 236-249.

Khoa, H.D.V., 2005. Modélisations des Glissements de Terrains Comme un Problème de Bifurcation. Ph.D. Thesis. Institut National Polytechnique de Grenoble.

Kozicki, J., Donzé, F.V., 2008. A new open-source software developed for numerical simulations using discrete modeling methods. Comput. Methods Appl. Mech. Eng. 197 (49-50), 4429-4443.

Kuhn, M.R., Chang, C.S., 2006. Stability, bifurcation, and softening in discrete systems: a conceptual approach for granular materials. Int. J. Solids Struct. 43 (20), 6026-6051.

La Ragione, L., Prantil, V.C., Sharma, I., 2008. A simplified model for inelastic behavior of an idealized granular material. Int. J. Plasticity 24 (1), $168-189$.

Lade, P.V., 1992. Static instability and liquefaction of loose fine sandy slopes. J. Geotech. Eng. ASCE 118, 51-71.

Lade, P.V., Pradel, D., 1990. Instability and flow of granular materials. I: Experimental observations. ASCE J. Eng. Mech. 116, $2532-2550$.

Li, M., Richmond, O., 1997. Intrinsic instability and nonuniformity of plastic deformation. Int. J. Plasticity 13 (8-9), 765-784.

Li, Y., Karr, D.G., 2009. Prediction of ductile fracture in tension by bifurcation, localization, and imperfection analyses. Int. J. Plasticity 25 (6), $1128-1153$.

Lignon, S., Laouafa, F., Prunier, F., Khoa, H.D.V., Darve, F., 2009. Hydro-mechanical modelling of landslides with a material instability criterion. Geotechnique 59 (6), 513-524.

Love, A.E.H., 1927. A Treatise of Mathematical Theory of Elasticity. Cambridge University Press, Cambridge.

Mandel, J., 1966. Cours de Mécanique des Milieux Continus, vol. 1. Gauthier Villars publ., Tome, pp. 82-83.

Neilsen, M.K., Schreyer, H.L., 1993. Bifurcations in elastic-plastic materials. Int. J. Solids Struct. 30 (4), 521-544.

Nicot, F., Darve, F., 2006. Micro-mechanical investigation of material instability in granular assemblies. Int. J. Solids Struct. 43, 3569-3595.

Nicot, F., Darve, F., 2007. A micro-mechanical investigation of bifurcation in granular materials. Int. J. Solids Struct. 44, 6630-6652.

Nicot, F., Darve, F., Khoa, H.D.V., 2007. Bifurcation and second-order work in geomaterials. Int. J. Numer. Anal. Methods Geomech. 31, $1007-1032$.

Nicot, F., Sibille, L., Darve, F., 2009. Bifurcation in granular materials: an attempt at a unified framework. Int. J. Solids Struct. 46, 3938-3947. 
Nicot, F., Darve, F., 2011. Diffuse and localized failure modes: two competing mechanisms. Int. J. Numer. Anal. Methods Geomech. 35 (5), $586-601$.

Petryk, H., 1993. Theory of bifurcation and instability in time-independent plasticity. In: Nguyen, Q.S. (Ed.), Bifurcation and Stability of Dissipative Systems, CISM Courses and Lecturers, vol. 327. Springer, pp. 95-152.

Plassiard, J.P., Belheine, N., Donzé, F.V., 2009. A spherical discrete element model: calibration procedure and incremental response. Granul. Matter 11 (5), 293-306.

Prunier, F., Nicot, F., Darve, F., Laouafa, F., Lignon, S., 2009a. 3D multiscale bifurcation analysis of granular media. J. Eng. Mech. (ASCE) 135 (6), 1-17.

Prunier, F., Laouafa, F., Lignon, S., Darve, F., 2009b. Bifurcation modeling in geomaterials, from the second-order work criterion to spectral analyses. Int. J. Numer. Anal. Meth. Geomech. 33 (9), 1169-1202.

Sibille, L., Nicot, F., Donzé, F.V., Darve, F., 2009. Analysis of failure occurrence from direct simulations. Eur. J. Environ. Civil Eng. 13, $187-201$.

Sibille, L., Donzé, F., Nicot, F., Chareyre, B., Darve, F., 2008. Bifurcation detection and catastrophic failure. Acta Geotec. 3 (1), 14-24.

Sibille, L., Nicot, F., Donze, F., Darve, F., 2007. Material instability in granular assemblies from fundamentally different models. Int. J. Numer. Anal. Methods Geomech. 31, 457-481.

Steinhauser, M.O., Grass, K., Strassburger, E., Blumen, A., 2009. Impact failure of granular materials - non-equilibrium multiscale simulations and highspeed experiments. Int. J. Plasticity 25, 161-182.

Tordesillas, A., Muthuswamy, M., 2009. On the modeling of confined buckling of force chains. J. Mech. Phys. Solids 57 (4), $706-727$.

Tordesillas, A., Walker, D.M., Lin, Q., 2010. Force cycles and force chains. Phys. Rev. E 81, 011302

Vardoulakis, I., Sulem, J., 1995. Bifurcation Analysis in Geomechanics. Chapman \& Hall Publisher, London.

Tsutsumi, S., Kaneko, K., 2008. Constitutive response of idealized granular media under the principal stress axes rotation. Int. J. Plasticity 24, 1967-1989.

Valanis, K.C., 1985. On the uniqueness of solution of the initial value problem in softening materials. J. Appl. Mech. 52 (3), 649-653.

Valanis, K.C., 1989. Banding and stability in plastic materials. Acta Mech. 77, 113-141.

Wan, R., Nicot, F., Darve, F., 2010. On the micromechanical formulation of stress dilatancy as a flow rule in plasticity. J. Eng. Mech., ASCE 136 (5), 589-598.

Weber, J., 1966. Recherches concernant les contraintes intergranulaires dans les milieux pulvérulents. Bull. Liaison Ponts-et-chaussées 20, 1-20.

Zhu, Q.Z., Shao, J.F., Mainguy, M., 2010. A micromechanics-based elastoplastic damage model for granular materials at low confining pressure. Int. J. of Plasticity 26 (4), 586-602. 